\title{
TITLE: \\ Dual Targeting of FLT3 and LSD1 Disrupts the MYC Super-Enhancer Complex in Acute Myeloid Leukemia.
}

\section{AUTHORS:}

William Yashar ${ }^{1,2,9}$, Brittany M. Smith ${ }^{1,2,9}$, Daniel J. Coleman ${ }^{1}$, Jake VanCampen ${ }^{1,2}$, Garth Kong $^{1,2}$, Jommel Macaraeg ${ }^{1,2}$, Joseph Estabrook ${ }^{3}$, Emek Demir ${ }^{2,4,5}$, Nicola Long ${ }^{1,6}$, Dan Bottomly ${ }^{1,7}$, Shannon K. McWeeney ${ }^{1,7}$, Jeffrey W. Tyner ${ }^{2,8}$, Brian J. Druker ${ }^{1,2,6}$, Julia E. Maxson $^{1,2}$, Theodore P. Braun ${ }^{1,2,6}$

${ }^{1}$ Knight Cancer Institute, Oregon Health \& Science University, Portland, OR, 97239, USA

2 Division of Oncologic Sciences, Department of Medicine, Oregon Health \& Science University, Portland, OR, 97239, USA

${ }^{3}$ Cancer Early Detection Advanced Research Center, Oregon Health \& Science University, Portland, OR, 97239, USA

${ }^{4}$ Department of Molecular and Medical Genetics, Oregon Health and Science University, 3181 SW Sam Jackson Park Rd, Portland, OR 97239, USA

${ }^{5}$ Pacific Northwest National Laboratories, 902 Battelle Blvd, Richland, WA 99354, USA

${ }^{6}$ Division of Hematology \& Medical Oncology, Department of Medicine, Oregon Health \& Science University, Portland, OR, 97239, USA

7 Division of Bioinformatics and Computational Biology, Department of Medical Informatics and Clinical Epidemiology, Oregon Health \& Science University, Portland, OR, 97239, USA

8 Department of Cell, Developmental \& Cancer Biology, Oregon Health \& Science University, Portland, OR, 97239, USA

9 These authors contributed equally

\section{CORRESPONDENCE:}

Theodore P. Braun

Knight Cancer Institute

3181 SW Sam Jackson Pk. Rd., KR-HEM

Portland, Oregon, 97239

braunt@ohsu.edu 


\begin{abstract}
Clinical responses to kinase inhibitor therapy in acute myeloid leukemia (AML) are limited by the inevitable development of resistance. A major contributor to resistance is early epigenetic adaptation, leading to persistence of a small number of leukemia cells. Here we show that inhibition of the epigenetic regulator lysine-specific demethylase 1 (LSD1) augments the response to inhibitors of the FLT3 kinase in AML. We demonstrate that combined FLT3 and LSD1 inhibition results in synergistic cell death of FLT3-mutant AML cells. The drug combination activates a pro-differentiative epigenetic and transcriptional program while simultaneously suppressing the activity of MYC target genes. High-resolution, multi-modal epigenetic analyses revealed that combined FLT3 and LSD1 inhibition results in the suppression of MYC-bound promoters and the activation of PU.1-bound enhancers. Regulon enrichment analysis in primary AML samples nominated STAT5 as a putative regulator of MYC gene expression. Inhibition of FLT3 results in a loss of STAT5 binding at the MYC blood super-enhancer and a loss of super-enhancer activation. In contrast, LSD1 inhibition prevents the removal of repressive H3K9me1 marks at MYC target genes, resulting in suppression of $M Y C$ expression. We validated these findings in 66 primary AML samples including 17 FLT3-ITD-positive AML samples, with the majority demonstrating improved responses with the drug combination. High MYC regulon activity was a predictor of response to the drug combination and RNA-seq on drug-treated AML samples revealed suppression of MYC target genes. Finally, single cell ATAC-seq on primary AML blasts treated ex vivo with the FLT3 and LSD1 inhibitor combination results in a shift from MYC super-enhancer-high to a MYC super-enhancer-low cell state. Collectively, these studies provide preclinical rationale for the investigation of dual FLT3 and LSD1 inhibition in a clinical trial.
\end{abstract}




\section{Introduction}

In patients with acute myeloid leukemia (AML), response to kinase inhibitor therapy is limited by the emergence of drug resistance ${ }^{1}$. While the development of drug resistance is a complex process with multiple co-existent mechanisms, epigenetic adaptation likely plays a prominent role ${ }^{2}$. The development of kinase inhibitor resistance through epigenetic adaptation is mediated by the persistence of a small population of cells that overcome initial drug exposure ${ }^{3}$. These remaining drug-tolerant persister cells ultimately give rise to a clone or clones harboring resistance mutations that prevent drug binding or activate a pathway that bypasses the targeted oncogenic kinase. One strategy to overcome resistance is to sequentially target emergent bypass pathways with kinase inhibitors ${ }^{4}$. However, studies in solid tumors, like EGFR-mutant lung cancer, suggest that this approach merely restarts the cycle of persistence-resistance and likely will not offer durable benefit ${ }^{5}$. A second approach is to target the mechanisms of persistence, which are believed to be driven by epigenetic changes. By selectively targeting the epigenetic mechanisms of cell survival during the early stages of drug response, durable responses to kinase inhibitors may be obtained by preventing the outgrowth of drug-resistant clones.

Mutations in FMS-like tyrosine kinase 3 (FLT3) occur in nearly a third of all AML cases and are associated with an inferior overall survival ${ }^{6}$. The most frequent mutation in FLT3 is the internal tandem duplication (ITD) of a juxta-membrane domain, enabling ligandindependent receptor activation and maintenance of downstream proliferative programs ${ }^{7}$. Multiple FLT3 kinase inhibitors have been evaluated clinically, with two earning FDA approval ${ }^{8,9}$. However, responses to these drugs are short-lived, limiting their use to patients who are ineligible for aggressive therapy or as adjuncts to conventional therapy. Therefore, we were interested in identifying approaches to deepen the initial response to FLT3 inhibitors with the goal of achieving longer-lasting clinical responses.

Epigenetic therapies have shown promise to potentiate the efficacy of kinase inhibitors in AML. Inhibitors of lysine-specific demethylase 1 (LSD1) have shown particular promise in reversing non-genetic mechanisms of resistance ${ }^{10}$. We have previously shown that inhibition of lysine-specific demethylase 1 (LSD1) augments the efficacy of JAK inhibitors in JAK/STAT-driven AML, driving synergistic repression of MYC target genes ${ }^{11}$. As the JAK/STAT signaling pathway is activated downstream from FLT3-ITD, we reasoned that LSD1 inhibition might also potentiate the efficacy of FLT3 inhibition in FLT3-ITD-positive $\mathrm{AML}^{7}$. Moreover, FLT3 mutations are the most common signaling mutations in AML and represent a logical initial cohort for early-stage clinical investigation of combined kinase and LSD1 inhibition.

Herein we report ex vivo drug screening data on a cell line model of FLT3-ITD AML and primary FLT3-ITD-positive AML samples demonstrating that LSD1 inhibition potentiates the efficacy of FLT3 inhibition. Using high-sensitivity epigenetic profiling with CUT\&Tag, we establish that FLT3 and LSD1 inhibitors act at distinct chromatin elements to suppress MYC activity and drive cell cycle exit. Using short-term ex vivo culture, we confirm that these transcriptional and epigenetic responses to combined FLT3 and LSD1 inhibition 
foccur in primary FLT3-ITD-positive leukemic blasts. Collectively, these data provide preclinical support for the clinical investigation of combined FLT3 and LSD1 inhibitors for patients with FLT3-mutant AML.

\section{Results}

Combined FLT3 and LSD1 inhibition results in synergistic repression of MYC target genes and synergistic activation of differentiation-associated genes

Our prior work suggests that the combination of kinase and LSD1 inhibition may be an effective therapeutic strategy in AML. To establish whether this approach is effective for FLT3-mutant AML, we treated several FLT3-ITD-positive (MOLM13 and MV4-11) and negative (K562) cell lines with multiple FLT3 and LSD1 inhibitors. In the FLT3-ITDpositive cell lines, we observed potent synergy between the FLT3 inhibitor, quizartinib, and the LSD1 inhibitor, GSK2879552 (Figure 1a; Supplementary Figure 1c). We saw similar synergy with a second FLT3 inhibitor, gilteritinib, as well as with a second LSD1 inhibitor, ORY-1001 (Supplementary Figure 1b, d, e). In contrast, minimal synergy was observed in the K562 FLT3-wild-type cell line (Supplementary Figure 1f-h), suggesting the activity of this drug combination has specificity for FLT3-mutant AML. In addition, we performed flow cytometry for Annexin $\mathrm{V}$ and PI staining to assess for apoptosis (Supplementary Figure 2a, b). Both early (Annexin V+/PI-) and late (Annexin V+/PI+) populations were increased with the drug combination as compared with single drug controls. To assess the toxicity to residual normal hematopoietic stem cells, we measured the impact of combined quizartinib and GSK2879552 on colony formation from healthy CD34+ cells (Supplementary Figure 2c). We observed minimal inhibition of colony formation with quizartinib, a modest reduction with GSK2879552, and no further decrease with the combination, suggesting the combination does not suppress hematopoietic stem cells function beyond that seen with either inhibitor alone. These results indicate that synergy exists between FLT3 and LSD1 inhibition in FLT3-ITD-positive AML.

LSD1 inhibitor monotherapy remodels the chromatin landscape by modulating gene regulatory networks, resulting in cell differentiation and death ${ }^{12-14}$. We therefore performed RNA-seq on MOLM13 cells treated with quizartinib, GSK2879552, or the combination for 24 hours. Unsupervised clustering of differentially expressed genes revealed two broad patterns of expression: genes that were synergistically upregulated by the drug combination and genes that were synergistically downregulated by the combination (Supplementary Figure 2d; Supplementary Table S1). Transcription factor target enrichment analysis showed an enrichment of MYC target genes in the clusters of synergistically downregulated genes (Figure 1b; Supplementary Figure 2e; Supplementary Table S2). MYC plays a central role in cell cycle and proliferation and is a key oncogenic driver of several myeloid leukemias including FLT3-ITD AML ${ }^{15,16}$. In contrast, a variety of transcription factor target gene sets were enriched in the genes upregulated by the drug combination, including SPI1/PU.1 targets. SPI1 encodes the transcription factor PU.1, which is critical for coordinating myeloid differentiation ${ }^{17}$. To further corroborate these gene expression profiles, gene set enrichment analysis (GSEA) was performed which revealed depletion of MYC target genes in cells treated with the drug combination (Figure 1c; Supplementary Table S3). Collectively, we observed that the combination activates an anti-proliferative 
and pro-differentiative transcriptional program that repressed MYC target genes and activates SPI1 target genes.

\section{Combined FLT3 and LSD1 inhibition drives differentiation and proliferative arrest through changes in the chromatin landscape}

A crucial component of LSD1-inhibitor activity has been ascribed to disruption of the LSD1 scaffolding function, loss of GFI1 binding to chromatin, and reactivation of enhancers associated with differentiation ${ }^{13}$. Therefore, to characterize the early chromatin dynamics after combined FLT3 and LSD1 inhibition, we utilized cleavage under targets and tagmentation (CUT\&Tag) to assess changes in acetylation of histone 3 lysine 27 (H3K27Ac), a marker of transcriptional activation ${ }^{18,19}$. We measured changes in H3K27Ac from MOLM13 cells, a cell line model of FLT3-ITD positive AML. After 2 hours of drug treatment, we identified 667 peaks of differential H3K27Ac read pileup. Unsupervised clustering of these regions revealed four clusters (Figure 1d; Supplementary Table S4). The peaks in cluster 1 showed synergistic repression of acetylation by the drug combination and were localized primarily to promoters (Figure 1e). The cluster 1 peaks were enriched for MYC motifs (Figure 1f), consistent with the findings of decreased gene expression of MYC target genes. An example of a synergistically downregulated peak is observed at the PVT1 promoter (Supplementary Figure 2f), a known MYC target gene and enhancer $^{20}$. Cluster 2 contained peaks with increased signal largely driven by LSD1 inhibition. Peaks in this cluster were nearly equally distributed at promoter and non-promoter elements and were enriched for GFI1/GFI1B motifs. Cluster 3 peaks were also localized at promoter and non-promoter elements and showed enrichment for RUNX1 motifs. RUNX1 is a critical regulator of myeloid differentiation and potentiates transcriptional activation of lineage-specifying transcription factors, including PU. $1^{21,22}$. An example of synergistically upregulated enhancers from clusters 2 and 3 are observed upstream of the lysozyme (LYZ) promoter, which is expressed in mature granulocytes (Supplementary Figure $2 \mathrm{~g}$ ). These data collectively show that the drug combination produces rapid changes to the chromatin landscape, resulting in decreased expression of cell cycle genes and an upregulation of differentiation-associated genes. These molecular programs appear to be controlled at distinct genomic loci with promoters and enhancers controlling the anti-proliferative and pro-differentiative effects of the drug combination respectively. Furthermore, based on pathway analysis and motif enrichment, MYC, PU.1, GFI1, and RUNX transcription factors are candidate regulators of these chromatin dynamics.

Chromatin segmentation reveals that MYC-, STAT5-, and PU.1-driven molecular programs underlie the response to combined FLT3 and LSD1 inhibition

Our CUT\&Tag results revealed significant changes in histone acetylation at both promoters and outside promoters in response to combined FLT3 and LSD1 inhibition, arguing that both types of putative regulatory elements have distinct roles in response to combined therapy. We therefore profiled a series of covalent histone marks in MOLM13 cells 6 hours following drug treatment, which enabled the segmentation of chromatin into promoters and enhancers, respectively. Trimethylation of histone 3 lysine $4(\mathrm{H} 3 \mathrm{~K} 4 \mathrm{me} 3)$ is primarily localized at promoters, whereas monomethylation of histone 3 lysine 4 $(\mathrm{H} 3 \mathrm{~K} 4 \mathrm{me} 1)$ is predominantly at enhancers ${ }^{23}$. We also profiled H3K27Ac at this same time 
point to understand transcriptional activation ${ }^{18,19}$ at prompters and enhancers. Following LSD1 inhibition, we observed regions of increased H3K4me3 and H3K4me1 levels (Supplementary Figure 3a-h), consistent with LSD1's known demethylase activity of histone 3 lysine $4^{13,24}$. Unsupervised clustering of regions of differential H3K27Ac signal at promoters and enhancers revealed multiple patterns of regulation (Figure 2a, b; Supplementary Table S5). Similar to the global 2-hour acetylation CUT\&Tag data from Figure 1, we observed a large cluster of repressed H3K27Ac signal at promoters that were enriched for MYC motifs along with other basic helix-loop-helix transcription factors, like CLOCK (cluster P2). These promoters were enriched for GO terms associated with cell cycle and proliferation (Supplementary Figure 3i; Supplementary Table S6). At enhancers, we observed an increase in H3K27Ac signal at enhancers enriched for SPI1/PU.1 motifs following treatment with the drug combination (cluster E4). These enhancers were enriched for GO terms associated with inflammatory signaling (Supplementary Figure 3j). Moreover, we observed suppression of H3K27Ac signal at enhancers enriched for FOS, MYB, and STAT motifs by the inhibitor combination (cluster E3). These transcription factors have all been implicated in proliferation of leukemia cells ${ }^{25-28}$. Collectively, this data suggests that combined FLT3 and LSD1 inhibition represses the activation of MYC-bound promoters associated with cell cycle and proliferation. Furthermore, these results implicate that dual FLT3 and LSD1 inhibition increases the activation of enhancers enriched for differentiation-associated transcription factor motifs and represses those enriched for with proliferation-associated transcription factors.

To better characterize the transcription factor regulators driving response to combined FLT3 and LSD1 inhibition, we profiled genome-wide binding of multiple candidate transcription factors following single or dual agent therapy using chromatin immunoprecipitation sequencing (ChIP-seq), cleavage under targets and release using nuclease (CUT\&RUN), and CUT\&Tag. We then examined the pileup of these factors at each cluster of differential H3K27Ac signal at promoters and enhancers (Figure 2e-f). We observed that there was modest enrichment of LSD1 at all acetylated promoter and enhancer regions, consistent with its role as a global chromatin regulator. MYC pileup was most pronounced at differential H3K27Ac promoters (cluster P2), and enhancers (cluster E3) suppressed by the combination. In both clusters, a loss of MYC signal was observed with combined FLT3 and LSD1 inhibition compared with no drug or single drug controls. STAT5 binding was localized to differential enhancers that were downregulated by quizartinib (clusters E1 and E3), which is consistent with studies demonstrating that STAT5 is a primary downstream target of FLT3 inhibitors ${ }^{27}$. At both sets of differential H3K27Ac enhancers, combined FLT3 and LSD1 inhibition (driven primarily by quizartinib) led to a global loss of STAT5 signal. Additional transcription factors implicated in the response to LSD1 inhibitors including RUNX1, CEBPA, PU.1, and GFI1 were next examined. While RUNX1 demonstrated global enrichment across all regions, CEBPA, PU.1, and GFI1 showed specific enrichment at differential enhancers that have increased $\mathrm{H} 3 \mathrm{~K} 27 \mathrm{Ac}$ signal with LSD1 inhibition (clusters E1 and E4). Notably, LSD1 inhibition led to a loss GFI1 signal at these clusters, consistent with previously reported displacement of GFI1 from chromatin and impediment of GFI1-mediated transcriptional suppression upon LSD1 inhibition $^{13}$. Collectively these results implicate MYC, STAT5, and GFI1 in the synergistic cytotoxicity of combined FLT3 and LSD1 inhibition. 


\section{Loss of MYC expression plays a key role in the response to combined FLT3 and LSD1 inhibition}

Our transcriptomic and epigenetic analyses nominated MYC as a key driver of the molecular responses to combined FLT3 and LSD1 inhibition. We observed that combined FLT3 and LSD1 inhibition results in the suppression in the total abundance of MYC transcript (Figure 3a) and in a genome-wide decrease in MYC binding (Figure 3b). We validated that the transcriptional suppression of MYC by the combination was associated with a decrease in MYC protein abundance (Supplementary Figure 4a). Modulation of MYC target genes can be influenced both by changes in MYC gene expression and activity. Oncogenic MYC is a key promoter of the cell cycle, modulating the activity of proteins that control its progression, like TP5329. MYC controls gene expression of cell cycle proteins through the recruitment of pause-released factors to poised RNA Polymerase II (RNA PollI), a key regulatory step in transcriptional control ${ }^{30}$. We observed that the drug combination significantly increases RNA Polll pausing at MYC-bound genes (Figure 3c; Supplementary Figure 4b; Supplementary Table S7), indicating a disruption in MYC's ability to promote RNA Polll pause release at its target genes. We therefore compared phosphoprotein levels of MOLM13 cells treated with the drug combination for 24 hours to those with no drug treatment. We identified causal interactions from changes in phosphoprotein levels using literature-derived gene regulatory networks and gene correlation data $^{31}$. We observed an increase in the protein levels of TP53 and an enrichment of a causal network controlled by TP53, consistent with repression of MYC cell cycle regulation (Supplementary Figure 4c; Supplementary Table S8). Together, these findings suggest a mechanism of combined FLT3 and LSD1 that suppresses MYC expression and activity.

To evaluate the importance of MYC expression to the mechanism of dual FLT3 and LSD1 inhibition, we derived a MOLM13 cell line with a doxycycline-inducible MYC expression construct. The construct enabled overexpression of $M Y C$ by approximately two-fold (Supplementary Figure 4d). MYC overexpression resulted in decreased sensitivity to the drug combination and shifted the point of maximum synergy, requiring 5 times the dose of quizartinib and GSK to achieve maximal effect (Figure 3d, e; Supplementary Figure 4e). Consistent with this, MYC overexpression also attenuated the induction of apoptosis by the drug combination (Supplementary Figure 4f). These data suggest that forced expression of MYC partially abrogates the effect of combined FLT3 and LSD1 inhibition and is important to the combination mechanism of action.

To identify potential regulators of $M Y C$ gene expression in AML that may mediate the response to the combination, we performed a regulon enrichment analysis using RNAseq on 681 primary AML samples. We generated a regulon enrichment score that reflected the predicted activity for 468 different transcription factors for each sample. Correlation of these transcription factor activity scores with $M Y C$ gene expression revealed a strong positive correlation between STAT5 regulon activity and MYC gene expression (Figure 3f; Supplementary Table S9). As FLT3 is a known activator of STAT5 ${ }^{27}$, we generated MOLM13 cell lines with doxycycline-inducible knockdown of STAT5 evaluate its 
role in the response to combined FLT3 and LSD1 inhibition. We characterized four hairpins, two theoretically targeting each isoform of STAT5 (STAT5A and STAT5B). We identified one hairpin that specifically targeted STAT5A, one that specifically targeted STAT5B, and one that targeted both A and B (Supplementary Figure 5a-c). The fourth hairpin was unable to reduce expression of either isoform to a substantial degree (data not shown). Knockdown of either isoform of STAT5 resulted in increased sensitivity to GSK2879552 as measured by a decrease in drug curve AUC (Figure 3g; Supplementary Figure 5d-f). Furthermore, we observed synergy between STAT5 knockdown and GSK2879552, demonstrating that a loss of STAT5 activity is sufficient to recapitulate a portion of the quizartinib effect (Figure 3h; Supplementary Figure 5g-i). Moreover, STAT5 knockdown resulted in reduced $M Y C$ expression, which was potentiated by the presence of GSK2879552 (Figure 3i; Supplementary Figure 5j, k). Furthermore, STAT5 knockdown augmented the ability of LSD1 inhibition to decrease expression of the MYC target gene GOS2 $^{32}$ and to upregulate the expression of protease ADAM23 associated with myeloid maturation $^{33}$. Collectively, these results suggest that the STAT5-MYC axis plays a major role in the response to dual FLT3 and LSD1 inhibition.

\section{Quizartinib regulates $M Y C$ expression via suppression of STAT5 binding to the MYC blood super-enhancer}

To identify the mechanism by which STAT5 regulates MYC expression in FLT3-mutant AML, we examined the binding data from our STAT5 ChIP-seq data. While we did not identify a STAT5 binding event at the MYC promoter, consistent with prior reports in other cell lines ${ }^{34}$, ranking STAT5 peaks by normalized read pileup revealed strong binding event 1.7 Mb downstream of MYC at the previously characterized MYC blood super-enhancer cluster (BENC) (Figure 4a; Supplementary Tables S10, 11) ${ }^{35}$. STAT5-bound elements within the MYC BENC, which is comprised of multiple distinct enhancer modules, showed a rapid decrease in H3K27Ac signal after treatment with either quizartinib or the drug combination (Figure 4b). Given the established role for the MYC BENC in regulating MYC gene expression during normal hematopoiesis, we hypothesized that this locus plays a role in the response to combined FLT3 and LSD1 inhibition. Therefore, we performed ATAC-seq to characterize the changes in activity of the MYC BENC and an upstream enhancer of MYC, PVT120. This analysis revealed a loss of chromatin accessibility across all modules in response to combination drug treatment (Figure 4c-e). Moreover, we observed signal of activating histone modifications $\mathrm{H} 3 \mathrm{~K} 27 \mathrm{Ac}$ and $\mathrm{H} 3 \mathrm{~K} 4 \mathrm{me} 1$ at the MYC BENC as well as candidate regulatory transcription factors MYC, PU.1, STAT5, GFI1, and LSD1. Evaluation of STAT5 binding profiles and H3K27Ac signal revealed several sub-modules that also display dynamic behavior in response to drug treatment but have not been previously characterized (A0.1-0.5, C1, G1). Of note, one of the downregulated enhancer regions identified in our differential enhancer activation analysis corresponded to BENC module C/D (Figure 2c). Collectively, our findings identify the downregulation of MYC gene expression as a key component of the response to dual FLT3 and LSD1 inhibition and nominate the MYC BENC as a crucial locus for this transcriptional regulation.

\section{LSD1 inhibition produces gene activation and repression at distinct genomic loci}


Our data demonstrate that dual FLT3 and LSD1 inhibition exert a portion of their activity via a STAT5-dependent decrease in MYC gene expression. However, MYC overexpression only partially reverses the impact of combination treatment, suggesting the involvement of additional mechanisms. Our data suggests that LSD1 inhibition may also exert a direct repressive effect on the expression of MYC target genes. In other cell types, LSD1 plays a role in gene activation via removal of repressive mono-/di-methylated histone 3 lysine 9 (H3K9me1/2) marks ${ }^{36}$. To evaluate this possible mechanism, we profiled the genome-wide distribution of H3K9me1 via CUT\&Tag (Figures 5a-c). Inhibition of LSD1, with or without inhibition of FLT3, resulted in an accumulation of H3K9me1 at MYC target genes co-bound with LSD1 (Figure 5d). This was accompanied by a loss of the reciprocal activating mark acetylated histone 3 lysine 9 (H3K9Ac; Supplementary Figure 6), consistent with the observed decrease in the expression of MYC target genes. LSD1 inhibition has been shown to interrupt transcriptional regulation by disrupting LSD1 scaffolding of GFI1 in the CoREST transcription repressor complex ${ }^{13}$. We found that GFI1 knockdown enhanced FLT3-inhibitor-dependent repression of MYC target genes (Supplementary Figure 7a-c) and increased sensitivity to FLT3 inhibition (Figure 5e). These results suggest that the repression of MYC target genes after LSD1 inhibition is driven, in part, via the accumulation of repressive H3K9me1 marks and disruption of GFI1/CoREST transcriptional regulation.

LSD1/PU.1 co-bound regions demonstrated distinct changes in H3K9me1 signal from LSD1/MYC co-bound regions. Of the 5,269 LSD1/PU.1 co-bound regions, 59.8\% $(3,152)$ of the regions overlapped with differential H3K27Ac enhancers defined in Figure 2 (Supplementary Figure 7d). In response to LSD1 inhibition, a small increase in H3K9me1 was observed as these loci, however signal returned to baseline with FLT3 inhibitor co-treatment, suggesting divergent regulatory pathways from MYC-bound regions (Supplementary Figure 7e, f). Consistent with the pattern of enhancer activation observed in prior studies, LSD1/PU.1 co-bound regions exhibited gains in activating H3K27Ac (Figure 5f) ${ }^{14}$. Prior studies have shown that PU.1 is necessary for LSD1-dependent enhancer activation and drug efficacy in MLL-driven AML cell lines ${ }^{12,13,13}$. Therefore, we assessed the impact of SPI1 knockdown on the efficacy of combined FLT3 and LSD1 inhibition. PU.1-deficient cells demonstrated no significant reduction in drug synergy (Figure $5 \mathrm{~g}$, h; Supplementary Figure $7 \mathrm{~g}-\mathrm{i}$ ). These findings suggest that dual inhibition of FLT3 and LSD1 exerts locusspecific effects on the chromatin landscape by interrupting GFI1/CoREST transcriptional regulation and altering the balance of $\mathrm{H} 3 \mathrm{~K} 9$ marks at MYC-bound promoters, while simultaneously facilitating gains in $\mathrm{H} 3 \mathrm{~K} 27 \mathrm{Ac}$ at enhancers (Figure $5 \mathrm{i}, \mathrm{j}$ ).

\section{Efficacy of combined FLT3 and LSD1 inhibition in primary AML samples}

We assessed the efficacy of combined FLT3 and LSD1 inhibition on 66 primary AML samples using a 3-day ex vivo drug assay (Supplementary Table S12). The LSD1 inhibitor GSK2879552 showed minimal single agent activity but augmented the efficacy of quizartinib in both FLT3-ITD-negative and -positive samples (Figure 6a, b; Supplementary Figure 8a). Similar to the pattern of synergy observed in cell lines, we found drug synergy across a broad range of doses and synergistic induction of apoptosis in primary AML blasts (Figure 6c; Supplementary Figure 8b, c). To further characterize the determi- 
nants of response to combined FLT3 and LSD1 inhibition, we performed a regulon enrichment analysis on baseline RNA-sequencing performed on the cohort (Figure 6d; Supplementary Table S13). Unsupervised clustering of the regulon enrichment signatures revealed a cluster constituted of $79.2 \%$ responders (19 of 24 patients) that showed upregulated regulatory activity of STAT5 and MYC. Responders are defined as samples that demonstrated a greater sensitivity to dual therapy than to either single agent. Moreover, MYC regulon activity was third strongest correlate with response to the combination (Figure 6e). These findings reveal that AML samples with high baseline MYC activity have the greatest sensitivity to combined FLT3 and LSD1 inhibition.

To characterize the dynamics of gene regulatory networks in response to combined FLT3 and LSD1 inhibition, we performed RNA-seq on 6 FLT3-ITD positive patient samples treated with FLT3 inhibitor, LSD1 inhibitor, or the combination. Unsupervised hierarchical clustering of differentially expressed genes across all 6 samples revealed a similar pattern to that observed in MOLM13 cells with both synergistically downregulated and upregulated patterns of expression (Figure 6f; Supplementary Table S14). MYC expression and regulatory activity were downregulated in all 6 samples by the drug combination, although differing patterns of individual drug effect were observed (Supplementary Figure 8d, e). Transcription factor target analysis again revealed suppression of MYC targets and activation of SPI1/PU.1 target genes (Figure 6g, h; Supplementary Table S15). Finally, GSEA revealed the drug combination decreased expression of MYC target genes and increased expression of differentiation-associated genes (Figure 6i, j; Supplementary Table S16). Collectively, this data validates the findings of MYC gene expression and regulon activity from AML cell lines in primary AML blasts.

\section{Dual FLT3 and LSD1 inhibition suppresses MYC super-enhancer activity in primary AML blasts}

Our initial investigations in AML cell lines demonstrate that suppression of chromatin accessibility at the MYC super-enhancer is an important mechanism of cytotoxicity produced by combined FLT3 and LSD1 inhibition. To validate this mechanism in primary AML blasts, we performed single cell ATAC-seq on primary AML blasts from three separate FLT3-ITD-positive patients after 24 hours of treatment with combined quizartinib and GSK2879552 or DMSO. Clustering and cell type identification by CD34+ expression revealed that all three samples were largely comprised of myeloid blasts (Figure 7a-c; Supplementary Figure 9a-c). Separation of cell clusters based on treatment revealed population shifts in response to treatment in all three samples (Figure 7d-f; Supplementary Figure 9d-f). Assessment of read pileup at the MYC BENC showed that these population shifts were associated with decreased chromatin accessibility at one or more BENC modules (Figure 7g-j; Supplementary Table S17). However, each sample showed a distinct pattern of change. Sample 2684 showed decreases in the majority of modules, 2645 demonstrated the most prominent decreases in modules $A$ and $G$, and sample 2603 the strongest decreases in modules F and G. Ranking of peaks in sample 2684 by $\log _{10}$ fold change in peak score between the drug combination and DMSO revealed that module $\mathrm{C}$ was amongst the regions with the greatest degree of change (Figure $7 \mathrm{k}$ ). Peaks with a negative $\log _{10}$ fold change are those whose peak score is suppressed following combined inhibition. Collectively, these results demonstrate that suppression of MYC BENC activity 
is a conserved feature of the response to dual FLT3 and LSD1 inhibition in primary AML samples. However, sample-to-sample heterogeneity does exist, suggesting diversity in the regulatory factors that sustain MYC BENC activity in primary AML samples.

\section{Discussion}

Activating mutations in FLT3 are amongst the most common molecular events in $\mathrm{AML}^{6}$. While FLT3 inhibitors are clinically available, they produce only modest improvements in survival $^{8,9}$. Here, we demonstrated that LSD1 inhibition potentiates the efficacy of FLT3 inhibition in FLT3-ITD AML cell lines and primary cell blasts. High-resolution transcriptomic and epigenetic profiling revealed that the mechanism of synergy is in part due to depletion of regulatory transcription factor binding, primarily STAT5, at the MYC BENC. Moreover, we identified additional evidence that dual FLT3 and LSD1 inhibition results in the accumulation repressive $\mathrm{H} 3 \mathrm{~K} 9$ me1 marks at MYC-controlled proliferation genes and activating H3K27Ac marks at PU.1-controlled differentiation genes. These findings provide rationale for the clinical investigation of combined FLT3 and LSD1 inhibitors for patients with FLT3-mutant AML and elucidates how epigenetic therapies augment the activity of kinase inhibitors in AML.

A crucial component to the mechanism of FLT3/LSD1 inhibitor synergy was altering MYC expression through regulation of the MYC BENC. Given MYC's central role in cancer cell proliferation, leukemia stem cell maintenance, and chemotherapy resistance ${ }^{37-41}$, it is an enticing target. However, designing direct inhibitors of MYC has proven challenging due to the absence of deep surface-binding pockets ${ }^{42,43}$.While other groups have demonstrated that $M Y C$ transcription can by altered by inhibiting regulators of this super-enhancer $^{44-48}$, regulation of MYC BENC activity by combined epigenetic modulatory drugs and kinase inhibition is a novel approach to targeting this central oncogenic regulator. Our single cell ATAC seq analysis revealed significant variation in the pattern of MYC BENC module utilization between AML samples at baseline and in response to drug treatment (Figure 7). Indeed, other studies have suggested that each BENC module is bound by a distinct set of transcription factors and regulates MYC expression in specific blood cell lineages ${ }^{49}$. How MYC module use varies between molecularly defined AML subtypes and how this relates to drug responses is an important question for future studies.

Prior work on LSD1 inhibitors has largely implicated the pro-differentiation effects of these drugs as the central mechanism of cytotoxicity. Consistent with prior studies, our work here shows that LSD1 inhibition strongly activates differentiation-associated enhancers which are bound by LSD1, PU.1 and GFI1 ${ }^{14}$. Prior work has shown that suppression of PU.1 expression results in a block in LSD1-inhibitor-induced differentiation and decreased cytotoxicity ${ }^{14}$. While our work confirmed the role of GFI1 as a putative mediator of LSD1-inhibitor responses, we found that PU.1 knockdown had little effect on the transcriptional or cytotoxic response to dual FLT3 and LSD1 inhibition (Figure 5; Supplementary Figure 7). A precise explanation for these disparate findings is unclear. Much of the prior work on LSD1 inhibition has been done in MLL-rearrangement-driven mouse and human cell lines, as LSD1 opposes the effects of MLL on H3K4 methylation ${ }^{12,50,51}$. It is therefore possible that this genetic background results in a differing dependence on PU.1 
for LSD1-inhibitor-dependent enhancer activation. Indeed, it is unclear whether the prodifferentiation transcriptional effects observed in our study are important to the drug effect which appears to be more dependent upon suppression of MYC expression and activity. Separation of these pro-differentiation and anti-proliferative effects of dual FLT3 and LSD1 inhibition will be an important question for future investigation.

Our data provides preclinical rationale for the efficacy of dual FLT3 and LSD1 inhibition in AML. Interestingly, our patient sample data suggests that the combination may have broad activity outside of FLT3-ITD AML. Indeed, the majority of AML samples remain cytokine-dependent for growth, and FLT3 inhibitors may target this necessary growth signal. Although we do not yet know whether the synergy we observed with the drug combination ex vivo will translate into more durable responses in patients, studies in other disease types suggest that treatments delivering deeper up-front responses often improve response duration ${ }^{52,53}$. Ultimately, this question will be best answered by evaluation in an early-stage clinical trial with accompanying monitoring of minimum residual disease.

\section{Methods}

\section{Cell and Patient Sample Culture}

\section{Cell Lines}

MOLM13 cells (DSMZ) were cultured in RPMI (Gibco) supplemented with $20 \%$ fetal bovine serum (FBS, HyClone), 2 mM GlutaMAX (Gibco), 100 units/mL Penicillin, and 100 $\mu \mathrm{g} / \mathrm{mL}$ Streptomycin (Gibco). MV-4-11 and K562 cells (ATCC) were cultured in IMDM supplemented with 10\% fetal bovine serum (FBS, HyClone), 2 mM GlutaMAX (Gibco), 100 units $/ \mathrm{mL}$ Penicillin, and $100 \mu \mathrm{g} / \mathrm{mL}$ Streptomycin (Gibco). All cells were cultured at $5 \% \mathrm{CO}_{2}$ and $37^{\circ} \mathrm{C}$. Cell lines were tested monthly for mycoplasma contamination.

\section{Patient Samples}

All patients gave informed consent to participate in this study, which had the approval and guidance of the institutional review boards at Oregon Health \& Science University (OHSU), University of Utah, University of Texas Medical Center (UT Southwestern), Stanford University, University of Miami, University of Colorado, University of Florida, National Institutes of Health (NIH), Fox Chase Cancer Center and University of Kansas (KUMC). Samples were sent to the coordinating center (OHSU; IRB\#9570; \#4422) ${ }^{13}$ where they were coded and processed. Mononuclear cells were isolated by Ficoll gradient centrifugation from freshly obtained bone marrow aspirates or peripheral blood draws. Clinical, prognostic, genetic, cytogenetic, and pathologic lab values as well as treatment and outcome data were manually curated from patient electronic medical records. Genetic characterization of the leukemia samples included results of a clinical deep-sequencing panel of genes commonly mutated in hematologic malignancies (Sequenome and GeneTrails (OHSU); Foundation Medicine (UTSW); Genoptix; and Illumina). Patient samples were cultured in RPMI with 10\% FBS and 10\% HS-5 conditioned media (ATCC) or SFEMII supplemented with 1x StemSpan CD34+ Expansion Media and $1 \mu$ M UM729 (StemCell Technologies). 


\section{Colony Assay}

Whole bone marrow was obtained (AllCells) and CD34+ cells were selected using CD34 MicroBead Kit (Miltenyi Biotec) according to manufacturer's instructions. For the colony assay, 500 CD34+ cells were used per replicate and plated in MethoCult ${ }^{\mathrm{TM}}$ H4435 Enriched (StemCell Technologies). The four groups were treated with quizartinib (1 $\mathrm{nM})$, GSK2879552 (100 nM), the combination, or DMSO. Plates were incubated for 14 days in $5 \% \mathrm{CO}_{2}$ and $37^{\circ} \mathrm{C}$. Samples were imaged using STEMvision (StemCell Technologies) and blinded prior to counting by another investigator by assigning letters randomly. ImageJ $(\mathrm{NIH})$ was used to count colonies after blinding.

\section{Drug Synergy}

Drug synergy was assessed using an $8 \times 8$ matrix of drug concentrations. Cells were treated for 72 hours prior to MTS assay to evaluate viability. Cell viability was used to calculate drug synergy with SynergyFinder based on the ZIP reference model ${ }^{54}$.

\section{RNA Interference}

Two SMARTvector Inducible shRNAs for Human SPI1 (V3IHSHER_10431275, V3IHSHER_10642739), two for STAT5A (V3IHSHEG_6691183, V3IHSHEG_4988581) two for STAT5B (V3IHSHER_4778243, V3IHSHER_6411380) and two for GFI1 (V3IHSHER_5266412 and V3IHSHER_5697821) in a hEF1a-TurboRFP or hEF1a-TurboGFP (STAT5A) backbone were obtained from Horizon Discovery. Both SPI1 shRNA construct showed effective knockdown of SPI1. STAT5A V3IHSHEG_6691183 was specific for STAT5A, STAT5A V3IHSHEG_4988581 was ineffective against either STAT5A or B, STAT5B V3IHSHER_6411380 was selective for STAT5B and V3IHSHER_4778243 knocked down both STAT5A and B. GFI1 V3IHSHER_5266412 produced effective GFI1 KD while V3IHSHER_5697821 was ineffective. Lentivirus was produced by transfecting Lenti-X 293T cells (Clontech) with the SMARTvector transfer plasmid and packaging/pseudotyping plasmids. psPAX2 was a gift from Didier Trono (Addgene plasmid \# 12260; http://n2t.net/addgene:12260; RRID:Addgene_12260) and pMD2.G was a gift from Didier Trono (Addgene plasmid \#12259; http://n2t.net/addgene:12259; RRID:Addgene_12259). The supernatants containing lentivirus was collected after 48 hours of culture and filtered with a 0.45 um filter. MOLM13 cells were transduced with virus via spinnoculation in the presence of polybrene. Transduced cells were selected with $1 \mu \mathrm{g} / \mathrm{mL}$ puromycin to produce a stable cell line.

\section{MYC Overexpression}

For human MYC overexpression, pDONR223_MYC_WT was a gift from Jesse Boehm \& Matthew Meyerson \& David Root (Āddgene plasmid \# 82927; http://n2t.net/addgene:82927; RRID:Addgene_82927) and cloned into pCW57.1, a gift from David Root (Addgene plasmid \#41393; http://n2t.net/addgene:41393; RRID:Addgene_41393). Lentiviral particles were generated as above and MOLM13 cells were selected after viral transduction with $1 \mu \mathrm{g} / \mathrm{mL}$ puromycin. After selection, cells were treated with $1 \mu \mathrm{g} / \mathrm{mL}$ doxycycline for 72 hours prior to experiments. 
Apoptosis was assessed 48-72 hours after drug treatment by flow cytometry using an eBioscience Annexin V -APC apoptosis detection kit (ThermoFisher) according to the manufacturer's instructions.

\section{Sequencing Methods}

Bulk RNA-Seq

MOLM13 cells were treated with 1 nM quizartinib, 100 nM GSK2879552, the combination, or equal volume of DMSO for $24 \mathrm{~h}$. Total RNA was isolated using a RNeasy Plus Mini Kit (Qiagen). BGI performed the library preparation and sequencing with 50BP SE sequencing. Patient samples were cultured in 10\% HS-5 CM/RPMI with $20 \% \mathrm{FBS}$ and treated with 500 nM quizartinib, 500 nM GSK2879552, the combination, or the equivalent volume of DMSO for 24 hours. Total RNA was isolated with RNeasy Micro kit (Qiagen) according to the manufacturer's instructions. Libraries were prepared using the NEBNext Low Input RNA Library Prep Kit for Illumina (NEB) according to the manufacturer's instructions. Libraries were sequenced by the OHSU Massively Parallel Sequencing Shared Resource (MPSSR) using 100 BP SE sequencing on an Ilumina NovaSeq S1 flow cell.

\section{Bulk ATAC-Seq}

MOLM13 cells were treated with 1 nM quizartinib, 100 nM GSK2879552, the combination, or an equivalent volume of DMSO for 24 hours. After treatment, 50,000 cells per replicate were harvested for Fast-ATAC sequencing performed as previously described ${ }^{55}$. In brief, cells were resuspended in cold PBS and tagmentation master mix (25 ul of 2x tagmentation buffer, 2.5 ul of TDE1 [Illumina], 0.5 ul of $1 \%$ digitonin; $2 x$ tagmentation buffer: $66 \mathrm{mM}$ Tris-Acetate, $\mathrm{pH} 7.8,132 \mathrm{mM}$ potassium acetate, $20 \mathrm{mM}$ magnesium acetate, 32\% v/v $\mathrm{N}, \mathrm{N}$-Dimethylformamide) was added. Samples were incubated at $37^{\circ} \mathrm{C}$ for 30 minutes. DNA was purified using Zymo Clean and Concentrator 5 Kit (Zymo). Transposed DNA was amplified and purified as described previously with adapted primers ${ }^{56,57}$. Samples were quantified using Qubit dsDNA HS Assay Kit (Invitrogen), pooled, and sequenced by BGI using 50 BP PE sequencing.

\section{CUT\&Tag}

MOLM13 cells were treated with 1 nM quizartinib, 100 nM GSK2979552, the combination, or an equal volume of DMSO for 2 or 6 hours. Benchtop CUT\&Tag was performed as previously described ${ }^{58}$. In brief, cells were counted, harvested, and centrifuged for $5 \mathrm{~min}$ at $300 \mathrm{xg}$ at room temperature. Cells were washed $2 \mathrm{X}$ in $1.5 \mathrm{~mL}$ wash buffer $(20 \mathrm{mM}$ HEPES pH 7.5, 150 mM NaCl, 0.5 mM Spermidine, 1x Protease inhibitor cocktail). Concanavalin A magnetic coated beads (Bangs Laboratories) were activated in binding buffer by washing 2X (20 mM HEPES pH 7.5, $10 \mathrm{mM} \mathrm{KCl,} 1 \mathrm{mM} \mathrm{CaCl}_{2}, 1 \mathrm{mM} \mathrm{MnCl}_{2}$ ). Washed cells were separated into 100,000 cell aliquots and $10 \mathrm{ul}$ of activated beads were added to each sample. Samples were rotated end-over-end for 7 minutes at room temperature. A magnetic stand was used to separate beads and the supernatant was removed. Primary antibody was diluted 1:50 in antibody buffer $(20 \mathrm{mM} \mathrm{HEPES} \mathrm{pH} \mathrm{7.5,150mM} \mathrm{NaCl,}$ $0.5 \mathrm{mM}$ Spermidine, 1× Protease inhibitor cocktail, 0.05\% digitonin, $2 \mathrm{mM}$ EDTA, 0.1\% BSA). The primary antibodies used were: H3K27Ac (ab4729, Abcam), H3K4me1 (\#5326, Cell Signaling Technologies), H3K4me3 (\#9751, Cell Signaling Technologies), RBP1 
(\#2629, Cell Signaling Technologies), H3K9me1 (\#C15410065, diagenode), H3K9Ac (\#C15410004, diagenode), CEBPA (\#8178, Cell Signaling Technologies), and Normal Rabbit IgG (\#2729, Cell Signaling Technologies as previously described ${ }^{58}$ ) was diluted 1:100 in dig-300 buffer (20 mM HEPES pH 7.5, $300 \mathrm{mM} \mathrm{NaCl}, 0.5 \mathrm{mM}$ Spermidine, 1x Protease inhibitor cocktail, $0.01 \%$ digitonin) and added to samples. Samples incubated for 1 hour at room temperature on nutator. Samples were washed $2 \mathrm{X}$ with dig-300 buffer then resuspended in tagmentation buffer (dig-300 buffer with $1 \mathrm{mM} \mathrm{MgCl}$ ). Samples were incubated at $37^{\circ} \mathrm{C}$ for 1 hour. DNA was extracted with phenol:chloroform extraction. Samples were amplified by PCR using custom Nextera primers at $400 \mathrm{nM}$ and NEBNext ${ }^{59}$. PCR conditions were set to: $72^{\circ} \mathrm{C}$ for 5 minutes, $98^{\circ} \mathrm{C}$ for 30 seconds, 14 cycles of $98^{\circ} \mathrm{C}$ for $10 \mathrm{sec}, 63^{\circ} \mathrm{C}$ for $10 \mathrm{sec}$, and $72^{\circ} \mathrm{C}$ for 1 minute. Libraries were purified with AMPure Beads (Beckman) and sequenced by the OHSU Massively Parallel Sequencing Shared Resource (MPSSR) on an llumina NovaSeq using 50 BP SE sequencing or NextSeq 500 using 37 BP PE sequencing.

\section{CUT\&RUN}

MOLM13 cells were treated with $1 \mathrm{nM}$ quizartinib, $100 \mathrm{nM}$ GSK2979552, the combination, or an equal volume of DMSO 24 hours. CUT\&RUN was performed as previously described $^{60}$. Briefly, concanavalin A magnetic coated beads (Bangs Laboratories) were washed $2 x$ in binding buffer (20 mM HEPES pH 7.5, $10 \mathrm{mM} \mathrm{KCl}, 1 \mathrm{mM} \mathrm{CaCl}, 1 \mathrm{mM}$ $\left.\mathrm{MnCl}_{2}\right)$ to activate. 500,000 cells per replicate were washed $2 x$ with wash buffer $(20 \mathrm{mM}$ HEPES pH 7.5, $150 \mathrm{mM} \mathrm{NaCl}, 0.5 \mathrm{mM}$ Spermidine, 1x Protease inhibitor cocktail). Cells were bound to beads by nutating for 10 minutes at room temperature. Cells were permeabilized and incubated overnight at $4{ }^{\circ} \mathrm{C}$ on nutator with primary antibody in antibody buffer (wash buffer, $0.001 \%$ digitonin, $3 \mathrm{mM}$ EDTA). The following antibodies were used at 1:50 PU.1 (MA5-15064, Invitrogen), GFI1 (ab21061, Abcam), and Normal Rabbit IgG (\#2729, CST). Bead slurry was washed $2 x$ with dig wash buffer (wash buffer, $0.001 \%$ dig) and resuspended with dig wash buffer and 1x pAG-MNase (Epicypher). Cell were incubated for 10 minutes on nutator at room temperature then washed $2 x$ with dig wash buffer followed by resuspension in pAG-MNase reaction mix (dig wash buffer, $2 \mathrm{mM} \mathrm{CaCl}$ ). Bead slurry was incubated for 2 hours at $4^{\circ} \mathrm{C}$ on nutator. STOP buffer $(340 \mathrm{mM} \mathrm{NaCl}, 20 \mathrm{mM}$ EDTA, $4 \mathrm{mM}$ EGTA, $50 \mu \mathrm{g} / \mathrm{mL}$ RNase A, $50 \mu \mathrm{g} / \mathrm{mL}$ glycogen, $0.02 \%$ dig) was then added, then tubes were incubated at $37^{\circ} \mathrm{C}$ for 10 minutes. DNA was extracted using phenol:cholorform extraction. Libraries were prepared using NEBNext Ultra II DNA Library Prep Kit (NEB), modified for CUT\&RUN as previously described ${ }^{61}$. After adapter ligation fragments were cleaned up with 1.75x AMPure beads (Beckman). Following PCR amplification, libraries were purified $2 x$ with $1.2 x$ AMPure beads to rid of adaptor fragments. Libraries were quantified on the 2100 Bioanalyzer instrument (Agilent) with the High Sensitivity DNA Analysis Kit (Agilent). Libraries were pooled and sequenced by MPSSR on a NextSeq 500 sequencer (Illumina) using 37 BP PE sequencing.

\section{ChIP-Seq}

ChIP-seq was performed using the SimpleChIP plus Enzymatic Chromatin IP Kit (Cell Signaling Technology). For each replicate, $2 \times 10^{7}$ cells were fixed in $4 \%$ formaldehyde (Sigma) for 10 minutes at room temperature then quenched with glycine, washed and 
stored at $-80^{\circ} \mathrm{C}$ until use. Nuclei were extracted according to the manufactures instructions and treated with 1.25 uL MNase in 500 uL Buffer B at $37^{\circ} \mathrm{C}$ for 20 minutes. Samples were sonicated on a Qsonic sonicator at $50 \%$ amplitude for 5 cycles of $15 \mathrm{sec}$ on $15 \mathrm{sec}$ off on ice. Crosslinks were reversed on a small aliquot of extracted chromatin quantified by $\mathrm{OD}_{260}$. A total of $5 \mu \mathrm{g}$ of chromatin was used for each immunoprecipitation. The following antibodies were used with a total of $5 \mu \mathrm{g}$ of each primary antibody used per immunoprecipitation: Rabbit anti-LSD1 (ab17721, Abcam), Rabbit ani-MYC (13987, Cell Signaling Technology), Rabbit anti RUNX1 (ab23980), Rabbit anti-STAT5 (94205S, Cell Signaling Technology) and Rabbit IgG (2729, Cell Signaling Technology). After overnight incubation, complexes were captured using protein $\mathrm{G}$ beads. Crosslinks were reversed and libraries prepped using an NEBNex Ultra II for DNA Library Prep kit. Libraries were sequenced by the OHSU Massively Parallel Sequencing Shared Resource (MPSSR). The STAT5 libraries were sequenced by Genewiz using a HiSeqX and 150 BP PE sequencing.

Reverse Phase Protein Array (RPPA)

MOLM13 cells were treated for 24 hours with $1 \mathrm{nM}$ quizartinib, 100 nM GSK2979552, the combination. Cells were washed $2 x$ in PBS then flash frozen. Cell pellets were lysed and processed by the University of Texas MD Anderson Cancer Center Functional Proteomics RPPA Core Facility.

Single Cell ATAC-Seq

Patient samples were treated with $500 \mathrm{nM}$ quizartinib and $500 \mathrm{nM}$ GSK2879552 or an equal volume of DMSO for 24 hours. Nuclei were prepared using the demonstrated protocol for primary cell nuclei extraction from 10x Genomics. ATAC libraries were prepared using Chromium Single Cell ATAC Library and Gel Bead kit v1.1 (10x Genomics, 1000176). Libraries were sequenced with 50 BP PE sequencing by the OHSU MPSSR.

\section{Primary AML Blast Dataset}

Gene mutation, drug response, and gene expression data from primary AML blasts was accessed through the BeatAML database ${ }^{6}$. Samples from collected from patients who were in remission or had MDS at the time of collection were excluded from downstream analysis. Samples with quizartinib/GSK2979552 single and dual agent drug response data were selected and stratified by FLT3-ITD mutation status. Gene expression dataset was downloaded in the form of RPKM.

\section{Data Analysis}

Bulk RNA-Seq

Raw reads were trimmed with Trimmomatic ${ }^{62}$ and aligned with STAR ${ }^{63}$. Two MOLM13 replicates were identified as outliers during QC and excluded from downstream analysis. Differential expression analysis was performed using DESeq2 ${ }^{64}$. Raw $p$ values were adjusted for multiple comparisons using the Benjamini-Hochberg method. GO analysis was performed using Enrichr ${ }^{65}$ and Gene Set Enrichment Analysis (GSEA) ${ }^{66}$. 
Regulon Enrichment Analysis

Regulon enrichment signatures were derived by comparing all gene expression features to the median expression level of all samples. Weights were assigned to the regulatory network and the positive and negative edges of each regulator were rank ordered. The first component of the enrichment signature, the local delta concordance signature, was derived by calculating the concordance between the magnitude of the weight assigned to a particular edge and the ranked position of that edge. The features associated with activation (positive edges within the regulatory network) were monotonically ranked from most lowly to highly expressed, whereas the features associated with repression (negative edges within the regulatory network) were ranked by a monotonically decreasing function. The second component of the enrichment signature, the local enrichment signature, was derived by capturing positional shifts in the local gene ranked signature and integrating those shifts with the weights assigned to overlapping features for a given regulon and the expression data set. The last component of the enrichment signature was derived by projecting the rank-sorted local signature onto the globally ranked feature space. A global enrichment signature from this projection was calculated for each regulator. The median of robust quantile-transformed ranked positions was used as the enrichment scores for both the local enrichment and global enrichment signatures. The median of robust quantile-transformed ranked positions for the three individual signatures were integrated and reported as single enrichment score for each regulator.

\section{Bulk ATAC-Seq Analysis}

ATAC libraries were aligned to the human genome (hg38) using BWA-MEM ${ }^{67}$ and sorted using SAMtools ${ }^{68}$. Duplicates were marked with Sambamba ${ }^{69}$, duplicates and mitochondrial reads were removed. Counts per million (CPM) normalized tracks were generated with deepTools ${ }^{70}$. Differential accessibility was assessed with DESeq2 ${ }^{64}$. GO analysis was performed using Genomic Regions Enrichment of Annotations Tool (GREAT) ${ }^{71}$. CPM normalized tracks were merged with bigWigMerge ${ }^{72}$ and visualized using Integrative Genomics Viewer ${ }^{73}$.

\section{CUT\&Tag and CUT\&RUN Analysis}

CUT\&Tag and CUT\&RUN libraries were aligned to the human genome (hg38) using Bowtie $2^{74}$ and the following options --local --very-sensitive-local --no-unal --no-mixed --nodiscordant --phred33 - $10-X 700$. Peaks were called using GoPeaks ${ }^{75}$ and the following option -mdist 1000 . High confidence peaks were defined by presence in $2 / 2$ or $2 / 3$ replicates. Consensus bed files were formed by merging the high confidence peaks from DMSO, quizartinib, GSK2979552, and the combination using BEDTools ${ }^{76}$. Differential peaks were identified using DESeq $2^{64}$ with default parameters. Heatmaps were produced using the ComplexHeatmap package from Bioconductor ${ }^{77}$. Peaks were annotated to the nearest feature using ChIPseeker ${ }^{78}$. GO analysis was performed using GREAT ${ }^{71}$. Counts tables for differential peaks were produced using multicov from BEDTools ${ }^{76}$. CPM normalized tracks, global signal heatmaps, and plot profiles at specified regions were generated using deepTools ${ }^{70}$. Active promoters were defined by the presence of H3K4me3 and H3K27Ac within $1000 \mathrm{bp}$ of a TSS. Active enhancers were defined by the presence of H3K4me1 and H3K27Ac beyond 1000 bp of a TSS. CPM normalized tracks were merged with bigWigMerge ${ }^{72}$ and visualized using Integrative Genomics Viewer ${ }^{73}$. Read 
pileup within $1500 \mathrm{~kb}$ upstream and downstream of LSD1-bound peaks as well as those that include or exclude MYC and PU.1 was quantified using computeMatrix referencepoint from deepTools ${ }^{70}$. Read pileup signal was scaled and visualized with a heatmap using the ComplexHeatmap package from Bioconductor ${ }^{77}$. RNA Polll pause indices were calculating by generating counts tables for each gene in a window from -30 to +100 around each TSS and then from the remainder of the gene bodies. Counts were divided by the region length and the pause index was calculated by dividing the TSS values by those for the corresponding gene body. Spearman correlation of transcription factor binding sites was assessed using multiBigwigSummary and plotCorrelation from deepTools ${ }^{70}$.

ChIP-Seq

ChIP-seq libraries were aligned to the human genome (hg38) using bowtie using the following options -v 2 -m 1 --best -strata. Peaks were called using MACS2 ${ }^{79}$ callpeak with the -narrow option and IgG library used as the control. Bigwigs for signal visualization were generated by sequentially converting alignments to bed files, big bed files then CPM normalized bigwig files using UCSC Kent Utilities. Read pileup at enhancers and promoters was evaluated using deepTools ${ }^{70}$. The STAT5 super-enhancer-like analysis was performed using the HOMER ${ }^{80}$ findPeaks -super command.

Reverse Phase Protein Array (RPPA)

The University of Texas MD Anderson Cancer Center Functional Proteomics RPPA Core Facility linearized, normalized, and batch-corrected (Level 4) the phosphoproteomic data. The data was analyzed with CausalPath ${ }^{31}(v$ 1.2.0) using a significant change of mean value transformation, an FDR threshold of 0.2 , and 1000 permutations for significance. The causal network was visualized on the CausalPath web server causalpath.org.

\section{Drug Response}

The percent change in quizartinib/GSK2979552 AUC with respect to quizartinib or GSK2979552 AUC was calculated using base R and visualized with ggplot2 (v 3.3.5). Responders were defined as samples whose percent change in combination-quizartinib $A U C$ is less than zero. The $R$ stats package ( $v$ 3.6.2) was used to calculate the spearman correlation between percent change combination-quizartinib AUC and regulon enrichment scores.

\section{Single Cell ATAC-Seq}

Single cell ATAC-seq libraries were aligned to the human genome (hg38) using 10x Genomics Cell Ranger ${ }^{81}$. ArchR ${ }^{82}$ was used in $\mathrm{R}$ to generate Arrow files for each fragment file, using a TSS enrichment of four and 5,000 unique fragments as cutoffs. An ArchRProject was created for each patient sample. Latent semantic indexing (LSI) was preformed using the addlterativeLSI function and clusters were added using the addClusters function. The UMAP for each patient sample, with both drug conditions integrated, was generated. The proportion of cells from each treatment condition was calculated and the dynamic CD34+ clusters were identified. Pseudo-bulk replicates from the dynamic clusters were normalized to TSS enrichment and used to generate bigwigs. The bigwigs were used to produce deepTools ${ }^{70}$ matrices to assess accessibility at the MYC BENC modules. The bar plots were generated using ggplot2. Peaks were called on pseudo-bulk replicates 
from the dynamic clusters using the ArchR ${ }^{82}$ addReproduciblePeakSet command and MACS2 ${ }^{79}$. Dynamic cluster peaksets from the same patient were combined using BEDTools ${ }^{76}$ intersect - loj command ${ }^{76}$. Peaks were ranked by their peak score and the $\log _{10}$ (peak score fold change) between dynamic clusters was calculated using a custom script.

\section{Quantification and Statistical Analysis}

Data are shown as mean \pm SEM. Prism software (version 9.1; Prism Software Corp.) or RStudio was used to perform statistical analysis, which are described in the figure legends. All data were analyzed with student t-test or ANOVA followed by Holm-Šidák correction. For differential analysis of RNA-seq, CUT\&Tag, CUT\&RUN, and ATAC-seq $p$ values were adjusted for repeated testing with a false discovery rate using the BenjaminiHochberg method ${ }^{83}$.

\section{Availability of data and materials}

The datasets supporting the conclusions of this article are available upon request.

\section{Acknowledgements}

We thank the following OHSU core facilities for their assistance: Advanced Light Microscopy, Flow Cytometry Shared Resource, Massive Parallel Sequencing Shared Resource, ExaCloud Cluster Computational Resource, and the Advanced Computing Center. Funding was provided by an American Society of Hematology Research Restart Award, an American Society of Hematology Scholar Award and 1 K08 CA245224 from NCl awarded to TPB. The Functional Proteomics RPPA Core is supported by MD Anderson Cancer Center Support Grant \# 5 P30 CA016672-40. WMY was supported by NIH T32 GM109835: Medical Scientist Training Program of Oregon Health and Science University.

\section{Author contributions}

WMY, BMS, TPB, DJC, BJD, and JEM designed the research plan. WMY, BMS, JM, DJC, and TPB performed the research. WMY, BMS, JV, GK, JM, DJC, JE, ED, and TPB contributed new reagents and/or analytic tools. WMY, BMS, TPB, BJD, and JEM analyzed the data. NL, DB, SKM, and JWT aided with analysis of BeatAML data. WMY and TPB wrote the manuscript. WMY, BMS, TPB, and JEM reviewed and edited the manuscript. The co-first authors may identify themselves as lead authors in their respective CVs.

\section{Competing Interests}

WMY potential competing interests -- is a former employee of Abreos Biosciences, Inc. and was compensated in part with common stock options. Pursuant to the merger and reorganization agreement between Abreos Biosciences, Inc. and Fimafeng, Inc., WMY surrendered all of his common stock options in 03/2021. JWT has received research support from Acerta, Agios, Aptose, Array, AstraZeneca, Constellation, Genentech, Gilead, Incyte, Janssen, Kronos, Meryx, Petra, Schrodinger, Seattle Genetics, Syros, Takeda, and Tolero. JEM -- SAB: lonis pharmaceuticals, Research Funding: Gilead Sciences. BJD potential competing interests -- SAB: Aileron Therapeutics, Therapy Architects (ALLCRON), Cepheid, Celgene, RUNX1 Research Program, Nemucore Medical Innovations, Novartis, Vivid Biosciences (inactive), Gilead Sciences (inactive); SAB \& Stock: 
Aptose Biosciences, Blueprint Medicines, EnLiven Therapeutics, Iterion Therapeutics, GRAIL, Recludix Pharma; Scientific Founder: MolecularMD (inactive, acquired by ICON); Board of Directors \& Stock: Amgen, Vincerx Pharma; Board of Directors: Burroughs Wellcome Fund, CureOne; Joint Steering Committee: Beat AML LLS; Founder: VB Therapeutics; Sponsored Research Agreement: EnLiven Therapeutics, Recludix Pharma; Clinical Trial Funding: Novartis, Astra-Zeneca; Royalties from Patent 6958335 (Novartis exclusive license) and OHSU and Dana-Farber Cancer Institute (one Merck exclusive license, one Cytolmage, Inc. exclusive license, and one Sun Pharma Advanced Research Company non-exclusive license); US Patents 4326534, 6958335, 7416873, 7592142, 10473667, 10664967, 11049247. The authors certify that all compounds tested in this study were chosen without input from any of our industry partners. The other authors do not have competing interests, financial or otherwise. 


\section{References}

1. Scholl, S., Fleischmann, M., Schnetzke, U. \& Heidel, F. H. Molecular Mechanisms of Resistance to FLT3 Inhibitors in Acute Myeloid Leukemia: Ongoing Challenges and Future Treatments. Cells 9, E2493 (2020).

2. Marine, J.-C., Dawson, S.-J. \& Dawson, M. A. Non-genetic mechanisms of therapeutic resistance in cancer. Nat Rev Cancer 20, 743-756 (2020).

3. Mullard, A. Stemming the tide of drug resistance in cancer. Nature Reviews Drug Discovery 19, 221-223 (2020).

4. Lovly, C. M. \& Shaw, A. T. Molecular Pathways: Resistance to Kinase Inhibitors and Implications for Therapeutic Strategies. Clin Cancer Res 20, 2249-2256 (2014).

5. Jackman, D. et al. Clinical Definition of Acquired Resistance to Epidermal Growth Factor Receptor Tyrosine Kinase Inhibitors in Non-Small-Cell Lung Cancer. Journal of Clinical Oncology (2009).

6. Tyner, J. W. et al. Functional genomic landscape of acute myeloid leukaemia. Nature 562, 526-531 (2018).

7. Kennedy, V. E. \& Smith, C. C. FLT3 Mutations in Acute Myeloid Leukemia: Key Concepts and Emerging Controversies. Frontiers in Oncology 10, 2927 (2020).

8. Stone, R. M. et al. Midostaurin plus Chemotherapy for Acute Myeloid Leukemia with a FLT3 Mutation. New England Journal of Medicine 377, 454-464 (2017).

9. Perl, A. E. et al. Gilteritinib or Chemotherapy for Relapsed or Refractory FLT3Mutated AML. New England Journal of Medicine 381, 1728-1740 (2019).

10. Bell, C. C. et al. Targeting enhancer switching overcomes non-genetic drug resistance in acute myeloid leukaemia. Nat Commun 10, 2723 (2019). 
11. Braun, T. P. et al. Combined inhibition of JAK/STAT pathway and lysine-specific demethylase 1 as a therapeutic strategy in CSF3R/CEBPA mutant acute myeloid leukemia. PNAS 117, 13670-13679 (2020).

12. Harris, W. J. et al. The Histone Demethylase KDM1A Sustains the Oncogenic Potential of MLL-AF9 Leukemia Stem Cells. Cancer Cell 21, 473-487 (2012).

13. Maiques-Diaz, A. et al. Enhancer Activation by Pharmacologic Displacement of LSD1 from GFI1 Induces Differentiation in Acute Myeloid Leukemia. Cell Rep. 22, $3641-3659$ (2018).

14. Cusan, M. et al. LSD1 inhibition exerts its antileukemic effect by recommissioning PU.1- and C/EBPa-dependent enhancers in AML. Blood 131, 1730-1742 (2018).

15. Call, S. G. et al. Targeting Oncogenic Super Enhancers in MYC-Dependent AML Using a Small Molecule Activator of NR4A Nuclear Receptors. Sci Rep 10, 2851 (2020).

16. Basit, F., Andersson, M. \& Hultquist, A. The Myc/Max/Mxd Network Is a Target of Mutated Flt3 Signaling in Hematopoietic Stem Cells in FIt3-ITD-Induced Myeloproliferative Disease. Stem Cells Int 2018, 3286949 (2018).

17. Nerlov, C. \& Graf, T. PU.1 induces myeloid lineage commitment in multipotent hematopoietic progenitors. Genes Dev 12, 2403-2412 (1998).

18. Creyghton, M. P. et al. Histone H3K27ac separates active from poised enhancers and predicts developmental state. Proc Natl Acad Sci U S A 107, 21931-21936 (2010).

19. Wang, Z. et al. Combinatorial patterns of histone acetylations and methylations in the human genome. Nat Genet 40, 897-903 (2008). 
20. Shigeyasu, K. et al. The PVT1 IncRNA is a novel epigenetic enhancer of MYC, and a promising risk-stratification biomarker in colorectal cancer. Mol Cancer 19, 155 (2020).

21. Imperato, M. R., Cauchy, P., Obier, N. \& Bonifer, C. The RUNX1-PU.1 axis in the control of hematopoiesis. Int J Hematol 101, 319-329 (2015).

22. Hu, Z. et al. RUNX1 regulates corepressor interactions of PU.1. Blood 117, 64986508 (2011).

23. Pundhir, S., Poirazi, P. \& Gorodkin, J. Emerging applications of read profiles towards the functional annotation of the genome. Front Genet 6, 188 (2015).

24. Shi, Y. et al. Histone Demethylation Mediated by the Nuclear Amine Oxidase Homolog LSD1. Cell 119, 941-953 (2004).

25. Kesarwani, M. et al. Targeting c-FOS and DUSP1 abrogates intrinsic resistance to tyrosine-kinase inhibitor therapy in BCR-ABL-induced leukemia. Nat Med 23, 472482 (2017).

26. Zhao, L., Ye, P. \& Gonda, T. J. The MYB proto-oncogene suppresses monocytic differentiation of acute myeloid leukemia cells via transcriptional activation of its target gene GFI1. Oncogene 33, 4442-4449 (2014).

27. Choudhary, C. et al. Activation mechanisms of STAT5 by oncogenic Flt3-ITD. Blood 110, 370-374 (2007).

28. Shahjahani, M., Abroun, A., Saki, N., Bagher Mohammadi, S. M. \& Rezaeeyan, H. STAT5: From Pathogenesis Mechanism to Therapeutic Approach in Acute Leukemia. Laboratory Medicine 51, 345-351 (2020). 
29. Meyer, N. \& Penn, L. Z. Reflecting on 25 years with MYC. Nat Rev Cancer 8, 976990 (2008).

30. Price, D. H. Regulation of RNA Polymerase II Elongation by c-Myc. Cell 141, 399400 (2010).

31. Babur, Ö. et al. Causal interactions from proteomic profiles: Molecular data meet pathway knowledge. Patterns (N Y) 2, 100257 (2021).

32. Heckmann, B. L., Zhang, X., Xie, X. \& Liu, J. The G0/G1 switch gene 2 (G0S2): regulating metabolism and beyond. Biochim Biophys Acta 1831, 276-281 (2013).

33. Zasłona, Z. et al. Transcriptome profiling of primary murine monocytes, lung macrophages and lung dendritic cells reveals a distinct expression of genes involved in cell trafficking. Respir Res 10, 2 (2009).

34. Pinz, S., Unser, S. \& Rascle, A. Signal transducer and activator of transcription STAT5 is recruited to c-Myc super-enhancer. BMC Molecular Biology 17, 10 (2016).

35. Bahr, C. et al. A Myc enhancer cluster regulates normal and leukaemic haematopoietic stem cell hierarchies. Nature 553, 515-520 (2018).

36. Metzger, E. et al. LSD1 demethylates repressive histone marks to promote androgen-receptor-dependent transcription. Nature 437, 436-439 (2005).

37. Kim, J. et al. A Myc network accounts for similarities between embryonic stem and cancer cell transcription programs. Cell 143, 313-324 (2010).

38. Nie, Z. et al. c-Myc is a universal amplifier of expressed genes in lymphocytes and embryonic stem cells. Cell 151, 68-79 (2012). 
39. Nanbakhsh, A. et al. c-Myc regulates expression of NKG2D ligands ULBP1/2/3 in AML and modulates their susceptibility to NK-mediated lysis. Blood 123, 3585-3595 (2014).

40. Fauriat, C. \& Olive, D. AML drug resistance: c-Myc comes into play. Blood 123, 3528-3530 (2014).

41. Li, L. et al. SIRT1 activation by a c-MYC oncogenic network promotes the maintenance and drug resistance of human FLT3-ITD acute myeloid leukemia stem cells. Cell Stem Cell 15, 431-446 (2014).

42. Yoshida, G. J. Emerging roles of Myc in stem cell biology and novel tumor therapies. J Exp Clin Cancer Res 37, 173 (2018).

43. Whitfield, J. R., Beaulieu, M.-E. \& Soucek, L. Strategies to Inhibit Myc and Their Clinical Applicability. Front Cell Dev Biol 5, 10 (2017).

44. Roe, J.-S., Mercan, F., Rivera, K., Pappin, D. J. \& Vakoc, C. R. BET Bromodomain Inhibition Suppresses the Function of Hematopoietic Transcription Factors in Acute Myeloid Leukemia. Mol Cell 58, 1028-1039 (2015).

45. Pelish, H. E. et al. Mediator kinase inhibition further activates super-enhancer-associated genes in AML. Nature 526, 273-276 (2015).

46. Zuber, J. et al. RNAi screen identifies Brd4 as a therapeutic target in acute myeloid leukaemia. Nature 478, 524-528 (2011).

47. Bhagwat, A. S., Lu, B. \& Vakoc, C. R. Enhancer dysfunction in leukemia. Blood 131, 1795-1804 (2018). 
48. Zhao, Y. et al. High-Resolution Mapping of RNA Polymerases Identifies Mechanisms of Sensitivity and Resistance to BET Inhibitors in t(8;21) AML. Cell Rep 16, 2003-2016 (2016).

49. Bahr, C. et al. A Myc enhancer cluster regulates normal and leukaemic haematopoietic stem cell hierarchies. Nature 553, 515-520 (2018).

50. Feng, Z. et al. Pharmacological inhibition of LSD1 for the treatment of MLLrearranged leukemia. J Hematol Oncol 9, 24 (2016).

51. Sakamoto, K. et al. Sensitivity of MLL-rearranged AML cells to all-trans retinoic acid is associated with the level of H3K4me2 in the RARa promoter region. Blood Cancer J 4, e205 (2014).

52. Branford, S. Why is it critical to achieve a deep molecular response in chronic myeloid leukemia? 1 105, 2730-2737 (2020).

53. Goldschmidt, H. et al. Deepening responses associated with improved progressionfree survival with ixazomib versus placebo as posttransplant maintenance in multiple myeloma. Leukemia 34, 3019-3027 (2020).

54. lanevski, A., Giri, A. K. \& Aittokallio, T. SynergyFinder 2.0: visual analytics of multidrug combination synergies. Nucleic Acids Res 48, W488-W493 (2020).

55. Corces, M. R. et al. Lineage-specific and single-cell chromatin accessibility charts human hematopoiesis and leukemia evolution. Nat Genet 48, 1193-1203 (2016).

56. Buenrostro, J. D., Wu, B., Chang, H. Y. \& Greenleaf, W. J. ATAC-seq: A Method for Assaying Chromatin Accessibility Genome-Wide. Current Protocols in Molecular Biology 109, 21.29.1-21.29.9 (2015). 
57. Buenrostro, J. D. et al. Single-cell chromatin accessibility reveals principles of regulatory variation. Nature 523, 486-490 (2015).

58. Kaya-Okur, H. S. et al. CUT\&Tag for efficient epigenomic profiling of small samples and single cells. Nature Communications 10, 1-10 (2019).

59. Buenrostro, J. D. et al. Integrated Single-Cell Analysis Maps the Continuous Regulatory Landscape of Human Hematopoietic Differentiation. Cell 173, 1535-1548.e16 (2018).

60. Skene, P. J. \& Henikoff, S. An efficient targeted nuclease strategy for high-resolution mapping of DNA binding sites. eLife 6, e21856 (2017).

61. Liu, N. et al. Direct Promoter Repression by BCL11A Controls the Fetal to Adult Hemoglobin Switch. Cell 173, 430-442.e17 (2018).

62. Bolger, A. M., Lohse, M. \& Usadel, B. Trimmomatic: a flexible trimmer for Illumina sequence data. Bioinformatics 30, 2114-2120 (2014).

63. Dobin, A. et al. STAR: ultrafast universal RNA-seq aligner. Bioinformatics 29, 15-21 (2013).

64. Love, M. I., Huber, W. \& Anders, S. Moderated estimation of fold change and dispersion for RNA-seq data with DESeq2. Genome Biology 15, 550 (2014).

65. Kuleshov, M. V. et al. Enrichr: a comprehensive gene set enrichment analysis web server 2016 update. Nucleic Acids Research 44, W90-W97 (2016).

66. Subramanian, A. et al. Gene set enrichment analysis: A knowledge-based approach for interpreting genome-wide expression profiles. PNAS 102, 15545-15550 (2005).

67. Li, H. \& Durbin, R. Fast and accurate short read alignment with Burrows-Wheeler transform. Bioinformatics 25, 1754-1760 (2009). 
68. Li, H. et al. The Sequence Alignment/Map format and SAMtools. Bioinformatics 25, 2078-2079 (2009).

69. Tarasov, A., Vilella, A. J., Cuppen, E., Nijman, I. J. \& Prins, P. Sambamba: fast processing of NGS alignment formats. Bioinformatics 31, 2032-2034 (2015).

70. Ramírez, F. et al. deepTools2: a next generation web server for deep-sequencing data analysis. Nucleic Acids Research 44, W160-W165 (2016).

71. McLean, C. Y. et al. GREAT improves functional interpretation of cis-regulatory regions. Nat Biotechnol 28, 495-501 (2010).

72. Kuhn, R. M., Haussler, D. \& Kent, W. J. The UCSC genome browser and associated tools. Briefings in Bioinformatics 14, 144-161 (2013).

73. Robinson, J. T. et al. Integrative Genomics Viewer. Nat Biotechnol 29, 24-26 (2011).

74. Langmead, B. \& Salzberg, S. L. Fast gapped-read alignment with Bowtie 2. Nat Methods 9, 357-359 (2012).

75. Yashar, W. M. et al. GoPeaks: Histone Modification Peak Calling for CUT\&Tag. [preprint] (2022) doi:10.1101/2022.01.10.475735.

76. Quinlan, A. R. \& Hall, I. M. BEDTools: a flexible suite of utilities for comparing genomic features. Bioinformatics 26, 841-842 (2010).

77. Gu, Z., Eils, R. \& Schlesner, M. Complex heatmaps reveal patterns and correlations in multidimensional genomic data. Bioinformatics 32, 2847-2849 (2016).

78. Yu, G., Wang, L.-G. \& He, Q.-Y. ChIPseeker: an R/Bioconductor package for ChIP peak annotation, comparison and visualization. Bioinformatics 31, 2382-2383 (2015). 
79. Zhang, Y. et al. Model-based Analysis of ChIP-Seq (MACS). Genome Biology 9, R137 (2008).

80. Heinz, S. et al. Simple combinations of lineage-determining transcription factors prime cis-regulatory elements required for macrophage and B cell identities. Mol Cell 38, 576-589 (2010).

81. Satpathy, A. T. et al. Massively parallel single-cell chromatin landscapes of human immune cell development and intratumoral T cell exhaustion. Nat Biotechno/ 37, 925-936 (2019).

82. Granja, J. M. et al. ArchR is a scalable software package for integrative single-cell chromatin accessibility analysis. Nat Genet 53, 403-411 (2021).

83. Johnson, W. E., Li, C. \& Rabinovic, A. Adjusting batch effects in microarray expression data using empirical Bayes methods. Biostatistics 8, 118-127 (2007). 

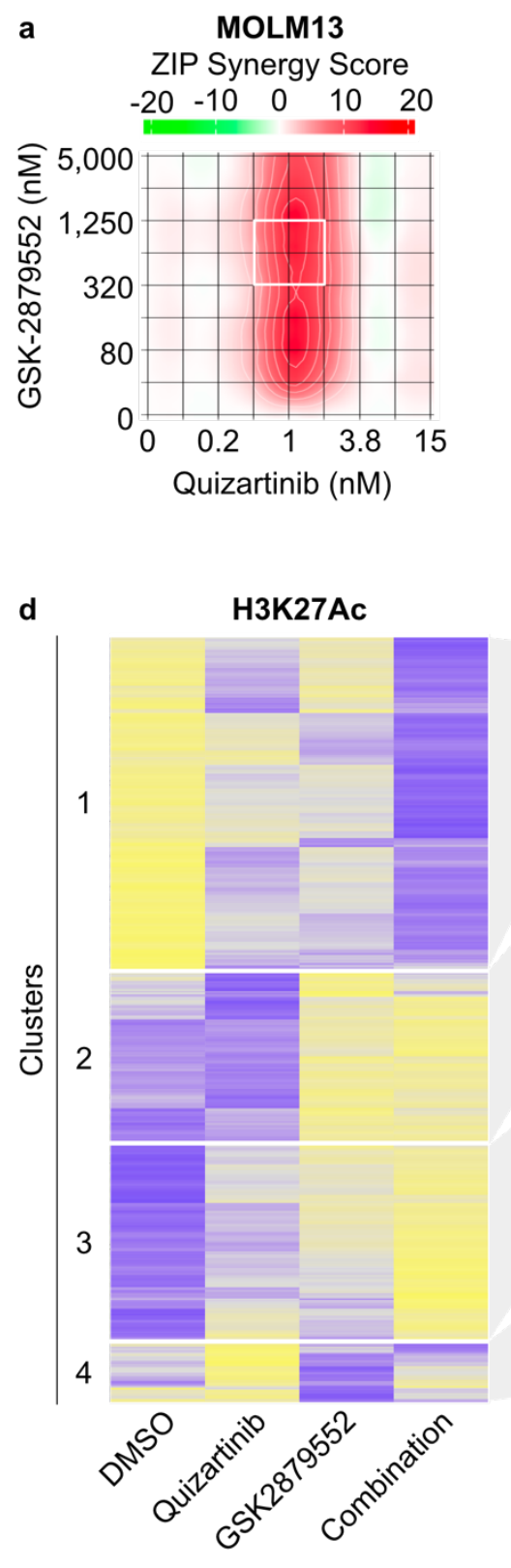
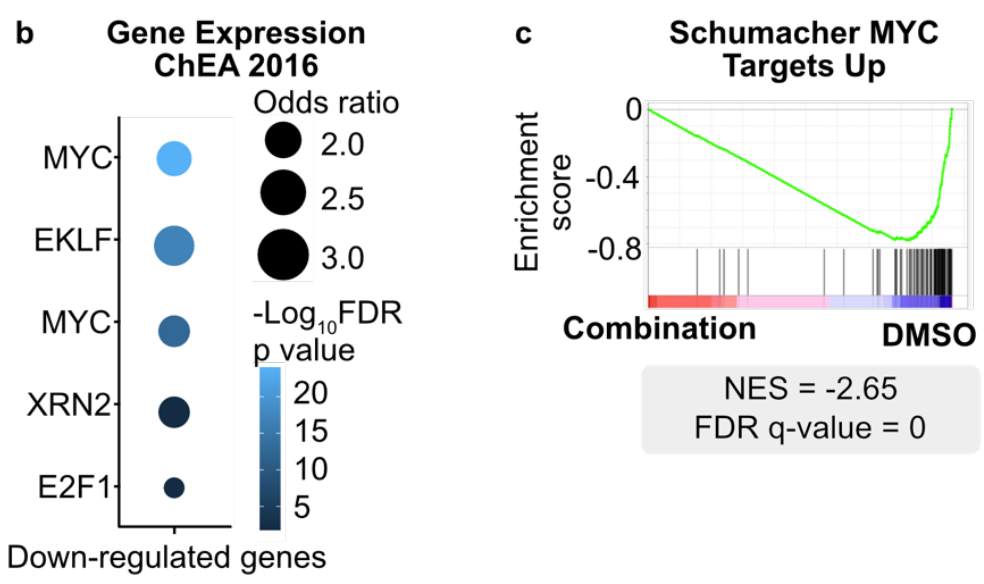
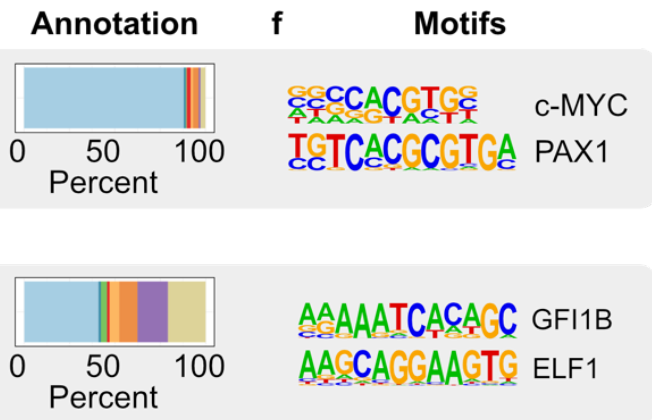

AAAAATCACACC GFI1B AACCAGGAAGTG ELF1

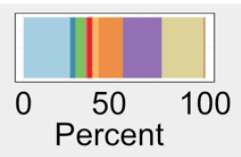

CTTAACCACAGA RUNX GAGACAGATITG ZBTB18

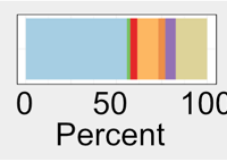

No Motifs

$$
\begin{aligned}
& \text { Scaled Feature } \\
& \text { counts Promoter }<1 \mathrm{~kb} \text { Other Exon } \\
& \text { Promoter 1-2 kb 1st Intron } \\
& \begin{array}{lllll}
2 & 0 & -2 & \begin{array}{l}
\text { Promoter 2-3 kb } \\
3^{\prime} \cup T R
\end{array} & \begin{array}{l}
\text { Other Intron } \\
\text { Distal Intergenic }
\end{array}
\end{array} \\
& \text { 1st Exon }
\end{aligned}
$$

Figure 1. Transcriptional and Chromatin Dynamics in Response to Combined FLT3 and LSD1 Inhibition in FLT3-ITD-positive AML.

a. MOLM13 cells were treated in triplicate with an $8 \times 8$ dose matrix of quizartinib and GSK2879552 for 3 days prior to viability assessment by CellTiter Aqueous colorimetric assay. Zero interaction potency (ZIP) synergy scores were calculated on the average values for each drug dose. b. MOLM13 cells were treated with quizartinib (1 nM), GSK2879552 (100 nM), the combination, or an equal volume of DMSO vehicle for 24 hours prior to RNA isolation and RNA sequencing. GO analysis was performed on genes whose expression significantly decreased with the combination relative to DMSO. c. GSEA was performed comparing the drug combination to DMSO. d. MOLM13 cells were 
treated with quizartinib (1 nM), GSK2879552 (100 nM), the combination, or an equal volume of DMSO vehicle for 2 hours prior to CUT\&Tag for H3K27Ac. Unsupervised hierarchical clustering of regions of differential read pileup with drug treatment. e. Annotation of peaks in clusters from d. f. Motif enrichment for regions of differential H3K27Ac read density. Top two de novo motifs with $p<10^{-12}$ shown. 

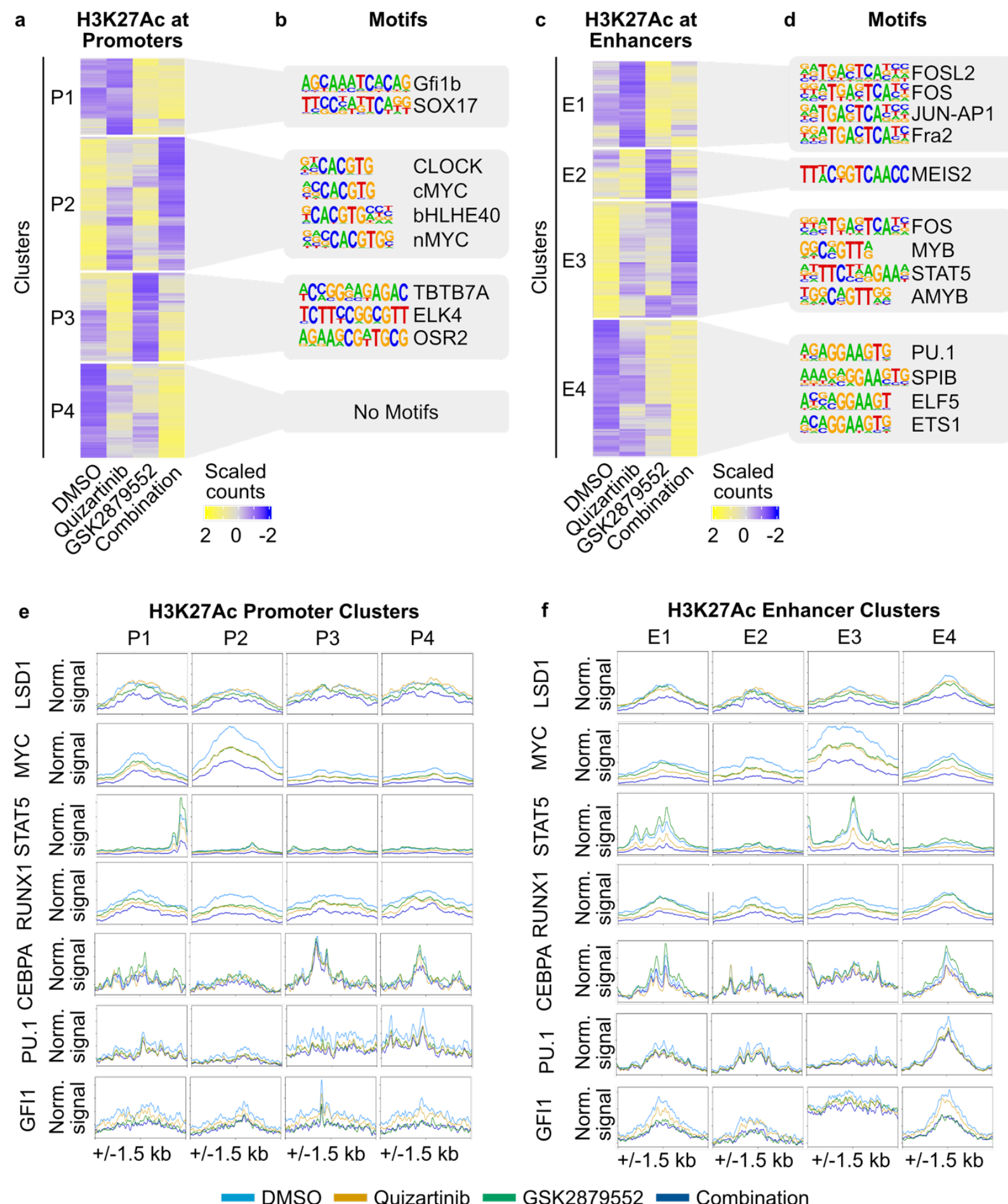

Figure 2. Discrete Components of the Response to FLT3 and LSD1 Inhibition are Mediated by Enhancers and Promoters.

a. MOLM13 cells were treated with quizartinib (1 nM), GSK2879552 (100 nM), the combination, or an equal volume of DMSO vehicle for 6 hours prior to CUT\&Tag for H3K27Ac, $\mathrm{H} 3 \mathrm{~K} 4 \mathrm{me} 1$, and $\mathrm{H} 3 \mathrm{~K} 4 \mathrm{me} 3$. On the basis of these marks, chromatin was segmented into enhancers and promoters. Unsupervised hierarchical clustering of differential H3K27Ac 
regions at promoters. b. Transcription factor motif enrichment is shown with top 4 known motifs. If no significant known motifs were identified, then de novo motifs with $p<10^{-12}$ are shown. c, d. Same analyses as a. and b. were performed at enhancers. e. MOLM13 cells were treated with quizartinib (1 nM), GSK2879552 (100 nM), the combination, or an equal volume of DMSO for 6 hours. LSD1, MYC, STAT5, and RUNX1 binding was assessed by ChIP-seq, CEBPA binding was assessed by CUT\&Tag, and GFI1 and PU.1 by CUT\&RUN. Transcription factor binding profiles at promoters with differential H3K27Ac identified in a. f. Transcription factor profiles at enhancers with differential H3K27Ac identified in $\mathrm{c}$. 


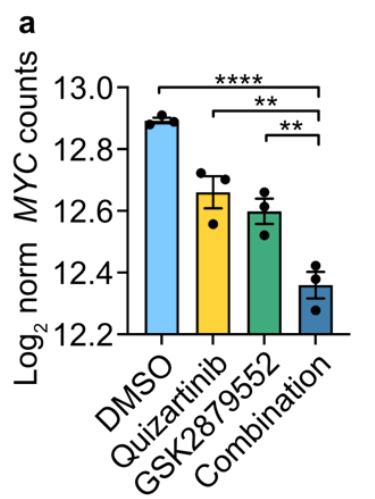

d
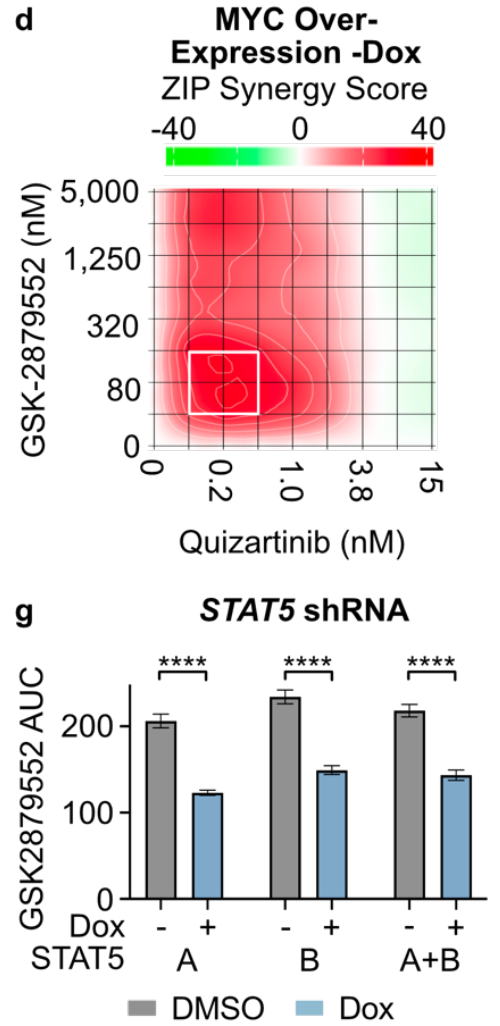

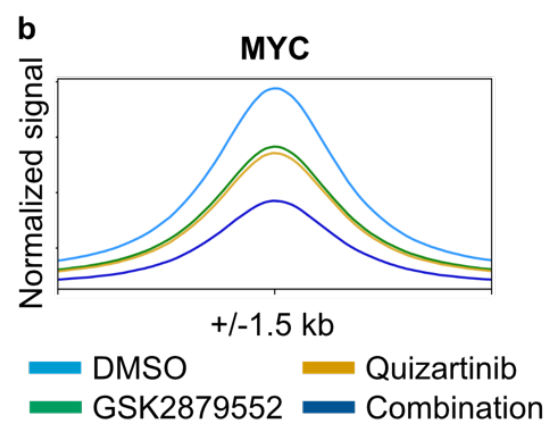

c MYC-Bound Regions

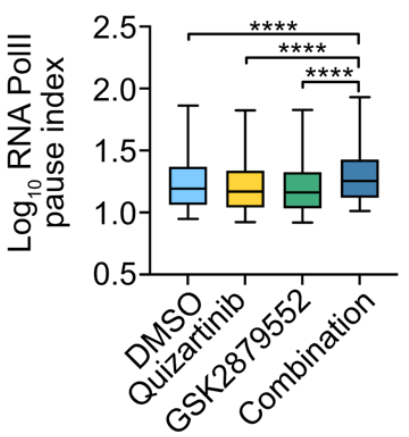

e

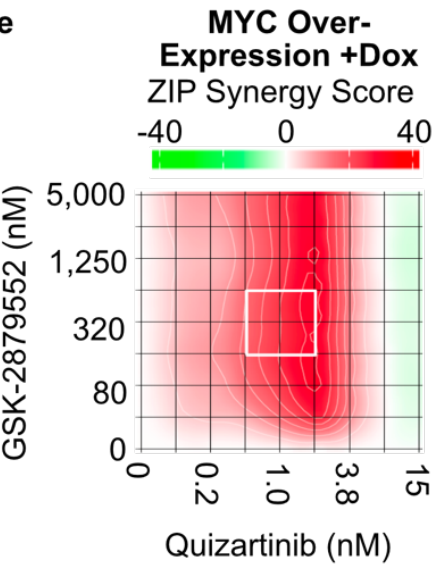

h

STAT5A+B ShRNA ZIP Synergy Score

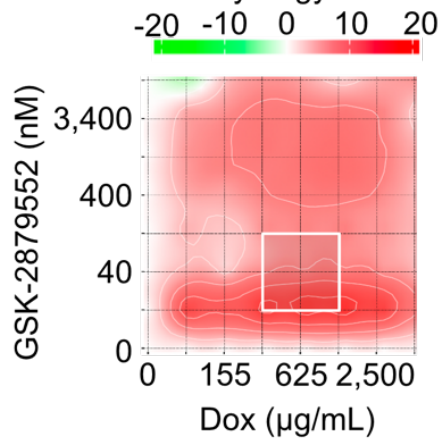

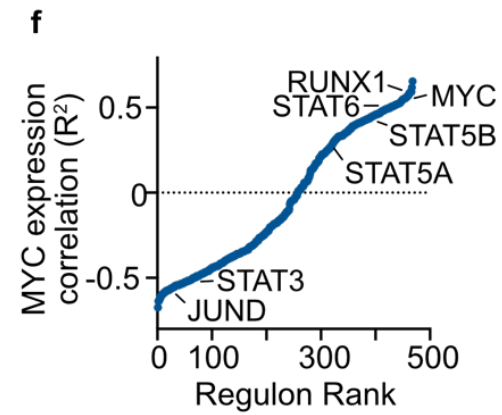

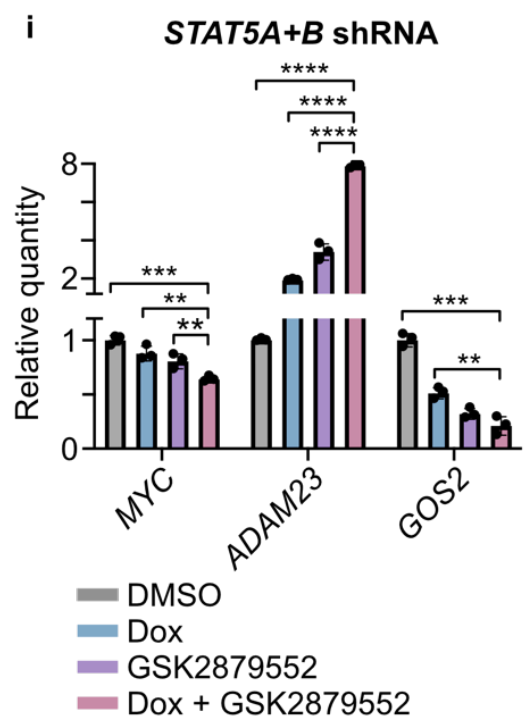

Figure 3. Combined FLT3 and LSD1 Inhibition Suppress MYC Expression by Altering Chromatin Dynamics at the MYC Super-Enhancer.

a. Normalized read counts for MYC from MOLM13 RNA-seq presented in Figure 1. Statistical significance was determined by two-way ANOVA with a Holm-Šidák post-test correction. b. MYC binding profile from MOLM13 ChIP-seq presented in Figure 2. c. MOLM13 cells were treated with quizartinib (1 nM), GSK2879552 (100 nM), the combination, or an equal volume of DMSO vehicle for 6 hours prior to CUT\&Tag for RBP1. RNA Polll pause-indices at RBP1 and MYC co-bound genes within the 25-95\% cumulative 
distribution. Statistical significance was determined by one-way ANOVA with a HolmŠidák post-test correction. d, e. MOLM13 cells harboring a doxycycline-inducible MYC construct were treated doxycycline $(1 \mu \mathrm{g} / \mathrm{mL})$ or an equivalent volume of DMSO for 72 hours then plated in triplicate in an $8 \times 8$ matrix of concentrations of quizartinib and GSK2879552 for 3 days. Cell viability was measured using CellTiter Aqueous colorimetric assay. Synergy was assessed using the ZIP method. f. Spearman's correlation of normalized MYC gene counts and regulon enrichment scores on RNA-seq performed in Figure 1. Regulons are ranked by goodness of fit $\left(R^{2}\right)$. g. MOLM13 cells were transduced with lentiviral particles harboring a doxycycline-inducible STAT5 knockdown. GSK2879552 AUC of cells treated with doxycycline $(1 \mu \mathrm{g} / \mathrm{mL})$ or DMSO for 72 hours. Statistical significance was determined by Student's t-test. $\mathbf{h}$. 8x8 drug synergy matrices between GSK2879552 and STAT5A+B knockdown (induced by doxycycline) were performed in triplicate in MOLM13 cells from $\mathrm{g}$. with viability assessed by CellTiter Aqueous colorimetric assay after 72 hours of drug exposure. Zero interaction potency (ZIP) synergy scores were calculated on the average values for each drug dose. i. qPCR assessment of gene expression in MOLM13 cells expressing a doxycycline-inducible STAT5A+B shRNA. Cells were treated with doxycycline $(1 \mu \mathrm{g} / \mathrm{mL})$ for 72 hours prior to the addition of GSK2879552 (100 nM) for 24 hours. Expression was normalized to GusB as an endogenous control. Statistical significance was determined by two-way ANOVA with a Holm-Šidák post-test correction. ${ }^{*}=p<0.05,{ }^{* *}=p<0.01,{ }^{* * *}=p<0.001,{ }^{* * * *}=p<$ 0.0001 . 

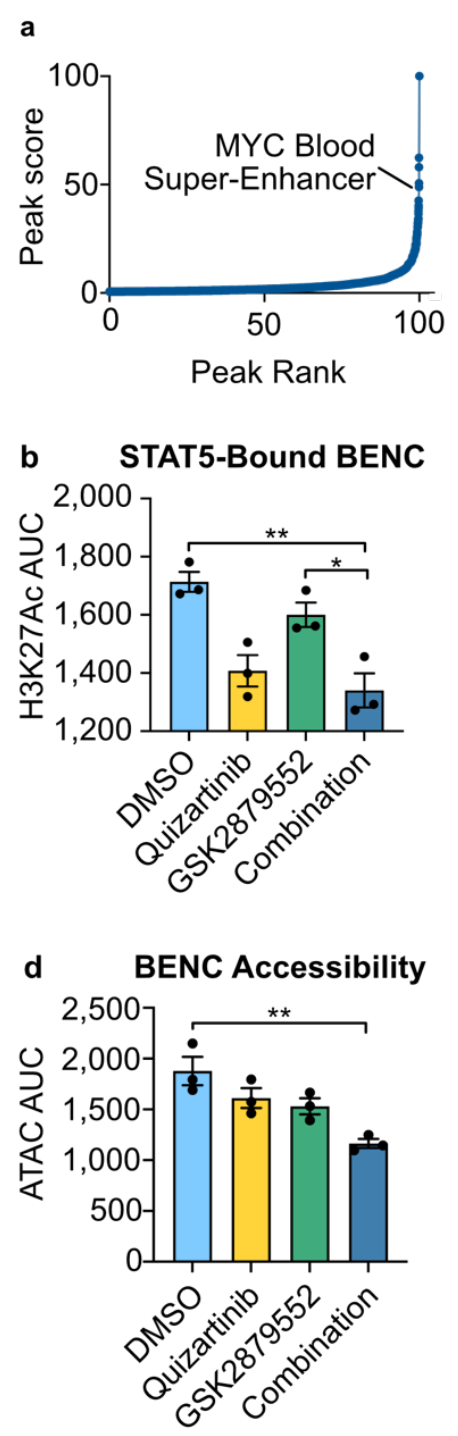

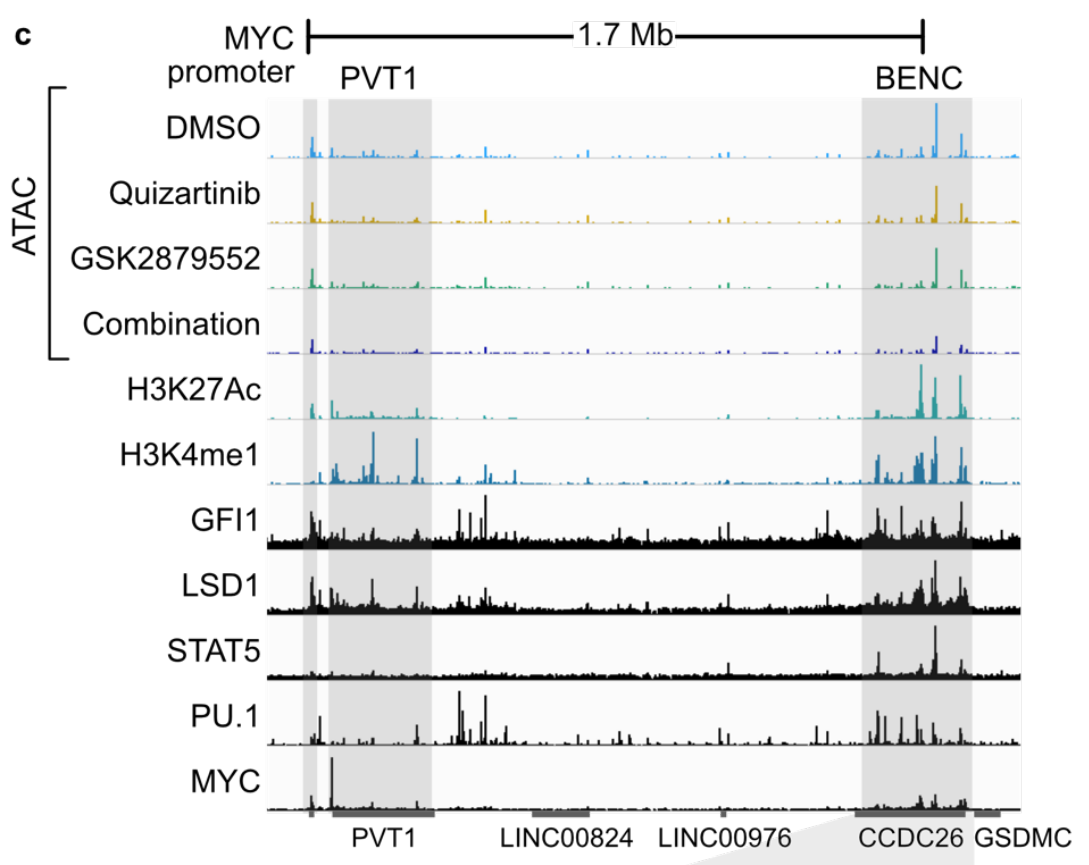

e $B E$

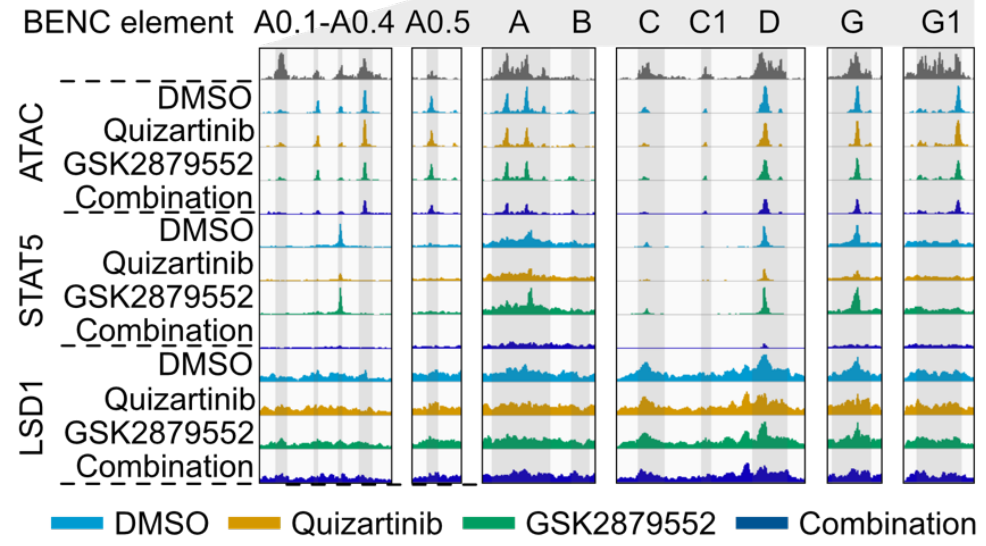

Figure 4. FLT3-Inhibition Represses MYC Expression Through a Loss of STAT5 Binding to the MYC Blood Enhancer Cluster.

a. STAT5 peak read pile up ranked by overall signal strength in MOLM13 cells from Figure 2. b. H3K27Ac signal AUC at STAT5-bound blood enhancer complex (BENC) elements. H3K27Ac signal from MOLM13 cells in Figure 1 whereas the STAT5 signal is from MOLM13 cells in Figure 2. Statistical significance was determined by two-way ANOVA with a Holm-Šidák post-test correction. c. ATAC-seq was performed on MOLM13 cells quizartinib (1 nM), GSK2879552 (100 nM), the combination, or an equal volume of DMSO vehicle for 24 hours. Representative histone modification and transcription factor tracks from MOLM13 cells shown at the extended MYC locus (same data from DMSO condition in Figure 2). d. ATAC signal AUC at all MYC BENC modules. Statistical significance was determined by two-way ANOVA with a Holm-Šidák post-test correction. e. ATAC, GFI1 CUT\&RUN, and STAT5 ChIP-seq signal at twelve independent BENC modules. ${ }^{*}=p<$ $0.05,{ }^{* *}=p<0.01,{ }^{* * *}=p<0.001,{ }^{* * * *}=p<0.0001$. 


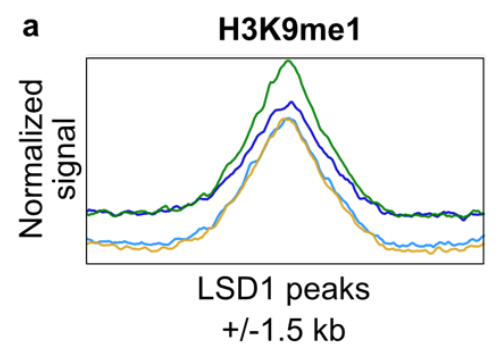

d

H3K27Ac

H3K9me1

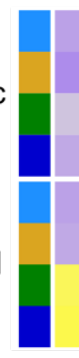

b

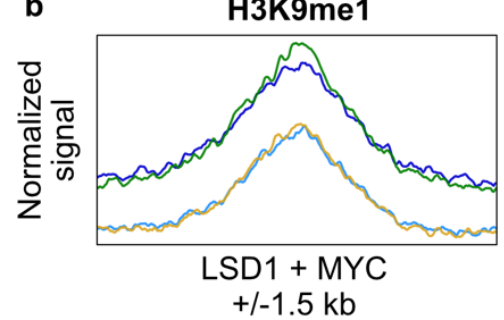

c $\quad$ H3K9me1

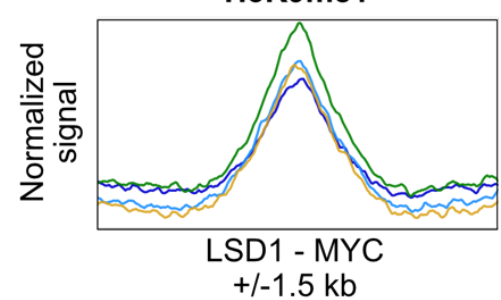

e GFI1 ShRNA

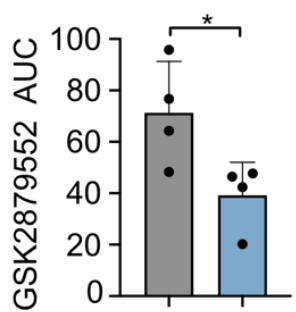

$0_{2} \rho_{0}$

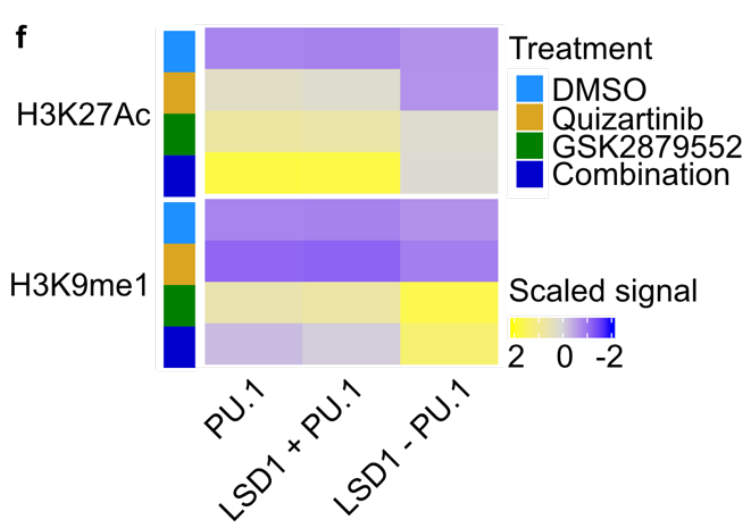

g SPI1 ShRNA 401 -Dox h ZIP Synergy Score

$\begin{array}{lllll}-40 & -10 & 0 & 10 & 40\end{array}$

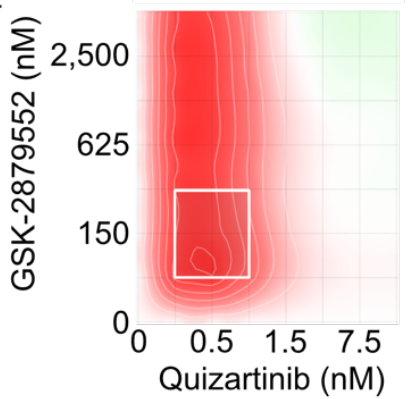

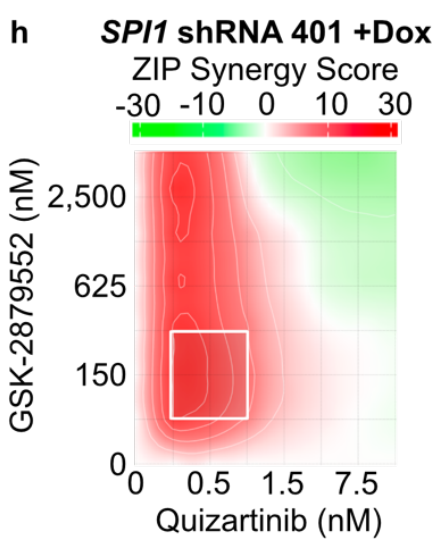
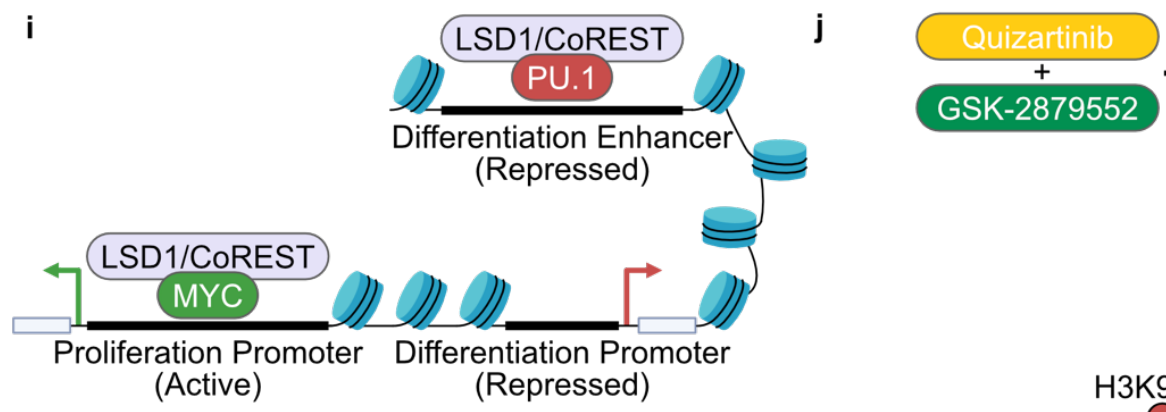

\section{LSD1/CoREST}
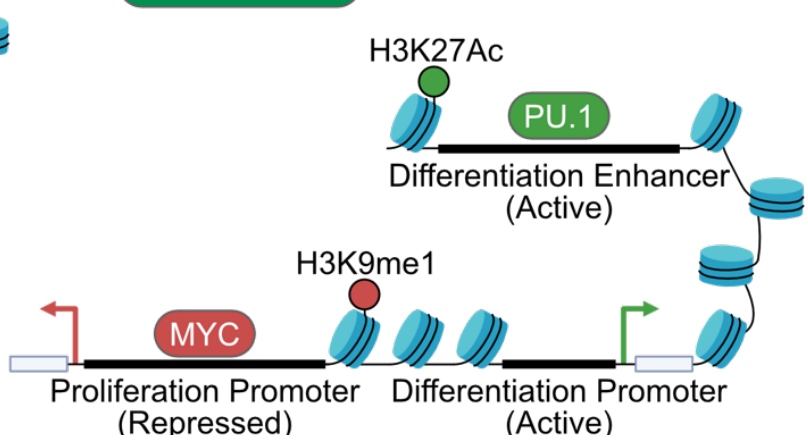

Figure 5. LSD1-Inhibition Induces a Gain of Inactivating H3K9me1 Binding at MYCbound Promoters and a Gain of Activating H3K27Ac Binding at PU.1-bound Enhancers. 
a-c. MOLM13 cells were treated with quizartinib (1 nM), GSK2879552 (100 nM), the combination, or an equal volume of DMSO vehicle for 6 hours prior to CUT\&Tag for H3K9me1. Normalized signal for H3K9me1 at regions of the genome bound by LSD1, at regions bound by both LSD1 and MYC from Figure 2, and at regions bound by LSD1 but not MYC. d. H3K27Ac and H3K9me1 signal at MYC-bound peaks, MYC/LSD1 co-bound peaks, and LSD1-bound peaks that include exclude MYC. e. GSK2879552 AUC in MOLM13 cells with or without GFI1 knockdown (induced by doxycycline). Statistical significance was determined by Student's t-test. ${ }^{*}=p<0.05$. f. H3K27Ac and H3K9me1 signal at PU.1-bound peaks, PU.1/LSD1 co-bound peaks, and LSD1-bound peaks that include exclude PU.1. g, h. 8x8 drug synergy matrices between GSK2879552 and quizartinib with and without SPI1 knockdown (induced by doxycycline) were performed in triplicate in MOLM13 cells with viability assessed by CellTiter Aqueous colorimetric assay after 3 days of drug exposure. Zero interaction potency (ZIP) synergy scores were calculated on the average values for each drug dose. i, j. Model describing the accumulation of H3K27Ac and H3K9me1 marks following dual FLT3 and LSD1 inhibition. LSD1, a member of the CoREST complex, directly removes repressive H3K9me1 and facilitates removal of activating $\mathrm{H} 3 \mathrm{~K} 27 \mathrm{Ac}$ via the recruitment of histone deacetylases. Dual FLT3 and LSD1 inhibition blocks this activity resulting in the repression of MYC-bound target genes associated with proliferation and the activation of PU.1-bound target genes associated with differentiation. 

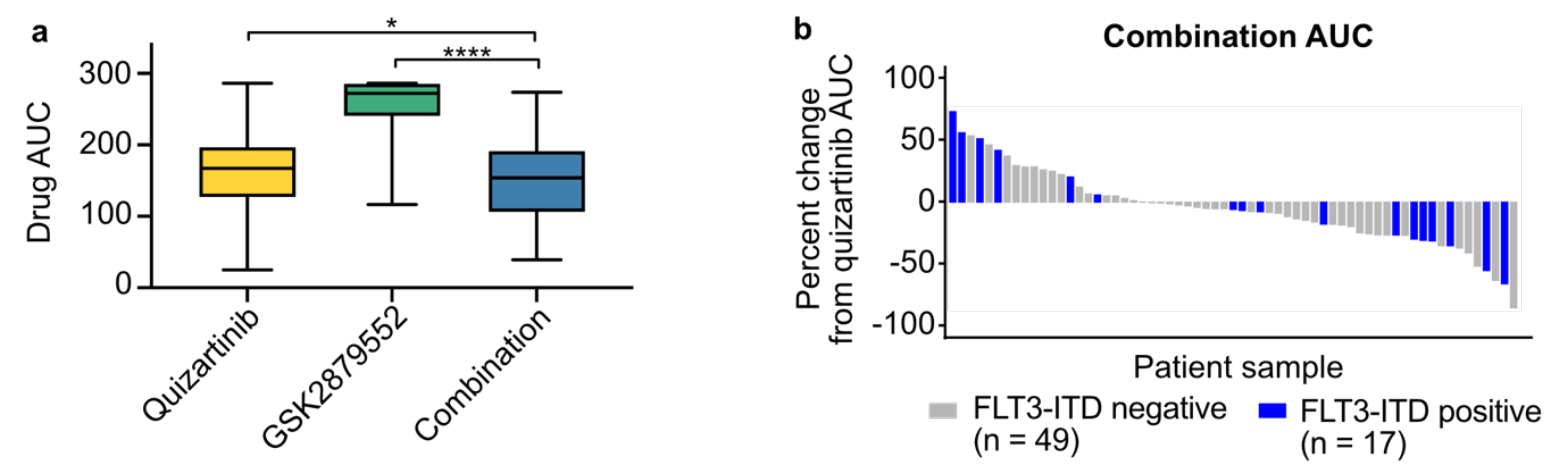

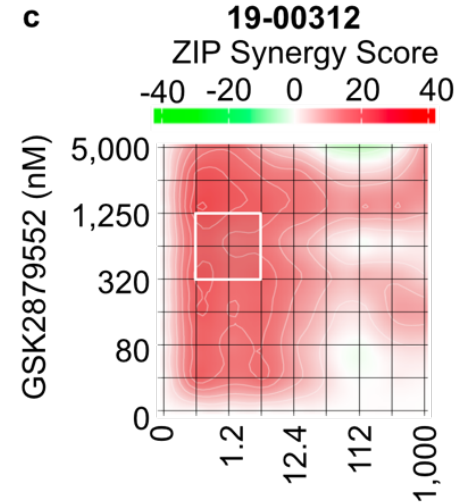

Quizartinib (nM)

f Gene Expression $(n=6)$
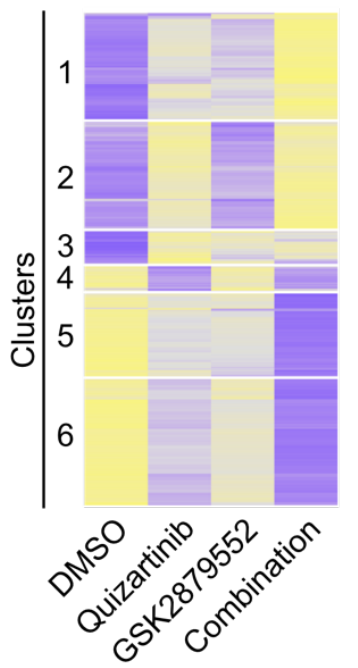

Scaled read counts

$\begin{array}{lll}2 & 0 & -2\end{array}$ d Patient Regulon Scores

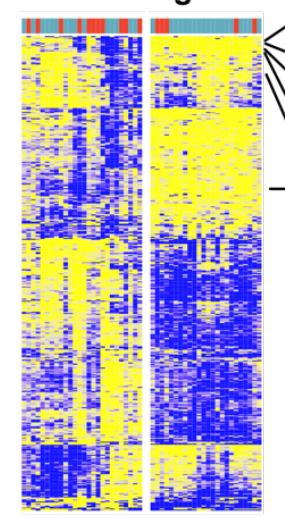

$<$ STAT5B

CDK8

MYCBP

MAZ

$-M Y C$

Scaled regulon score

$\begin{array}{llll}2 & 0 & -2\end{array}$

Response

Responder

Non-Responder

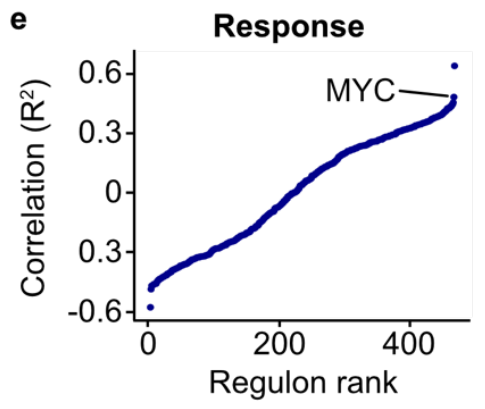

g Gene Expression Clusters 1/2 ChEA 2016

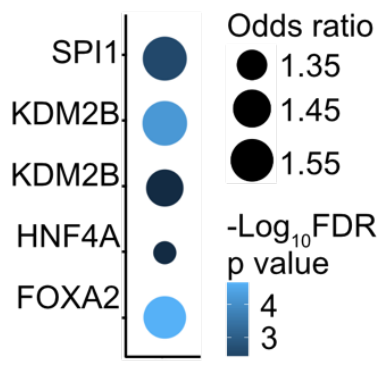

i

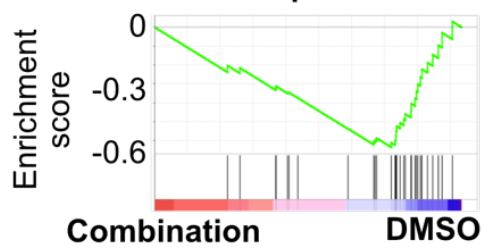

NES $=-1.9$

FDR q-value $=0.155$ h Gene Expression Clusters 5/6 ChEA 2016

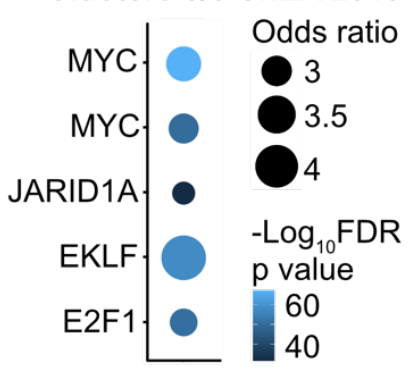

j $\begin{gathered}\text { Myeloid Cell } \\ \text { Development }\end{gathered}$

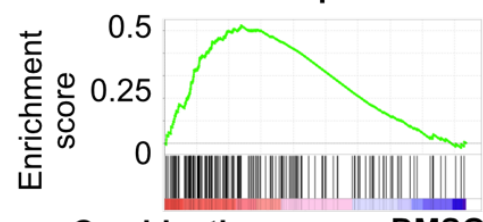

Combination DMSO

NES $=1.9$

FDR q-value $=0.025$

Figure 6. Combined FLT3 and LSD1 Inhibition Drives Synergistic Cell Death by Repressing a MYC-Dependent Transcriptional Network in Primary AML Blasts.

a. Primary AML blasts from 66 total samples (17 FLT3-ITD-positive) were cultured for 3 days along a 7-point curve with either quizartinib, GSK2879552, or equimolar amounts of 
the drug combination. Cell viability was assessed by CellTiter Aqueous colorimetric assay. Drug AUC for each single drug or the combination is shown. Statistical significance was determined by two-way ANOVA with a Holm-Šidák post-test correction. b. Percent change in combination AUC with respect to quizartinib AUC for each sample. Bar color indicates FLT3-ITD mutation status. c. Primary blasts from a FLT3-ITD-positive AML sample were treated in triplicate with an 8x8 dose matrix of quizartinib and GSK2879552 for 3 days prior to viability assessment by CellTiter Aqueous colorimetric assay. Zero interaction potency (ZIP) synergy scores were calculated on the average values for each drug dose. d. Regulon enrichment analysis on bulk RNA-seq performed on FLT3-ITDpositive AML samples classified as responders and non-responders to the drug combination. Regulons of interest are noted. e. Spearman's correlation of percent change in quizartinib and combination AUC in b, which was used to determine response, and regulon enrichment scores in d. f. Bulk RNA-seq was performed on six independent, FLT3ITD-positive patient samples treated in triplicate with $500 \mathrm{nM}$ quizartinib, $500 \mathrm{nM}$ GSK2879552, both drugs in combination, or an equivalent volume of DMSO for 24 hours. Unsupervised hierarchical clustering of genes differentially expressed upon drug treatment. g, h. Transcription factor target enrichment from clusters in f. i, j. Select GSEA plots from RNA-seq in $\mathrm{f}$. 
a

a $\quad \begin{array}{r}2684 \\ \quad \text { Single Cell ATAC }\end{array}$

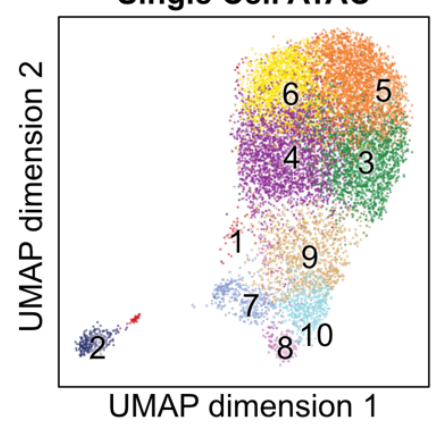

d

Cluster Composition

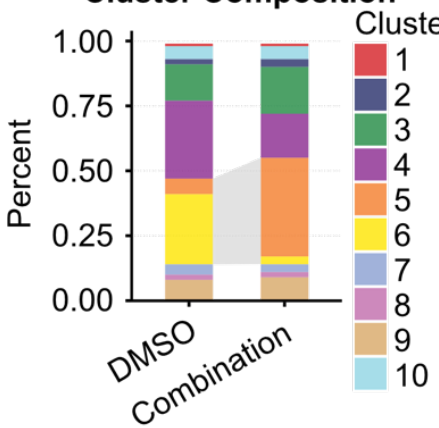

g Clusters 5/6

Accessibility at BENC

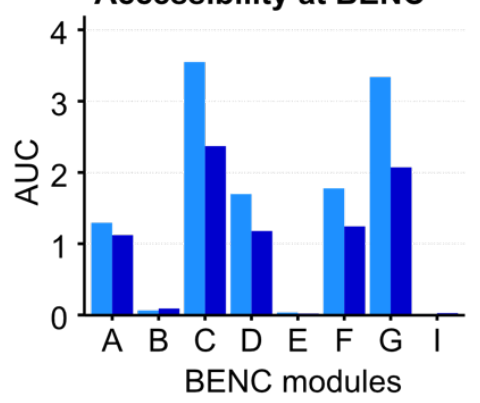

DMSO

Combination b
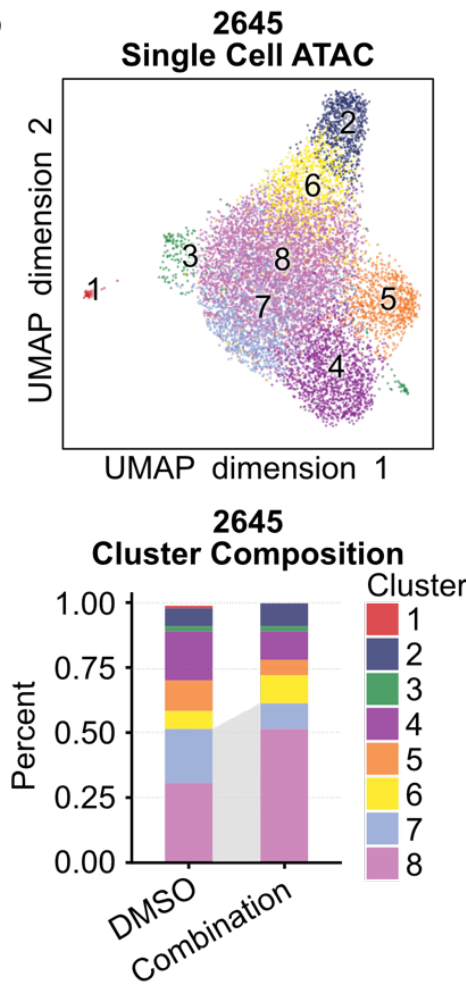

h

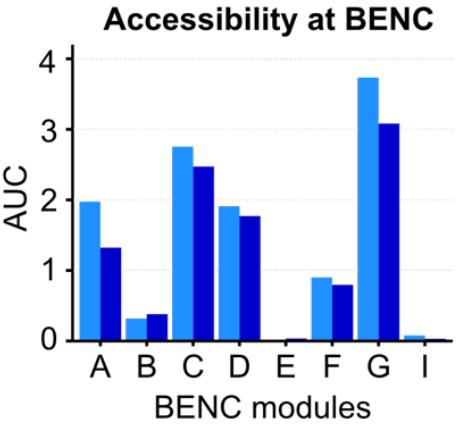

DMSO Combination
C
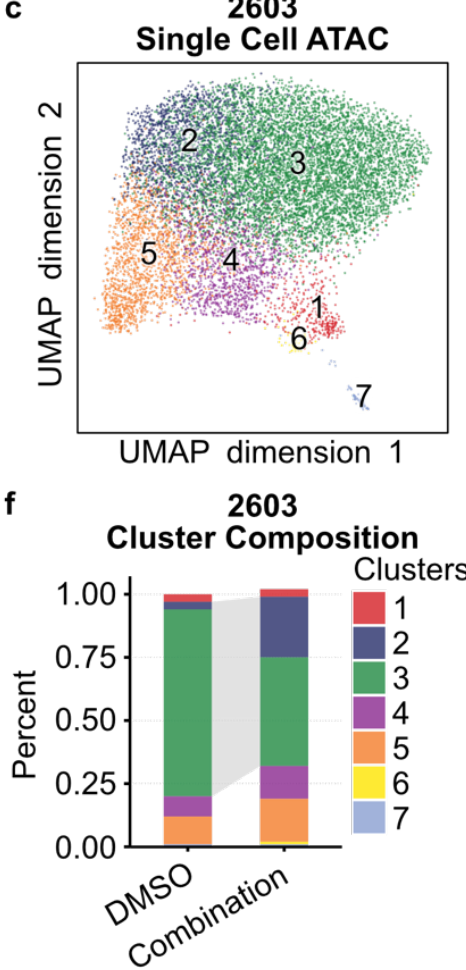

Clusters $2 / 3$

Accessibility at BENC

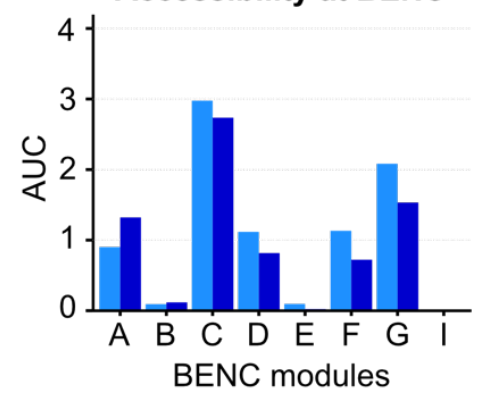

DMSO Combination

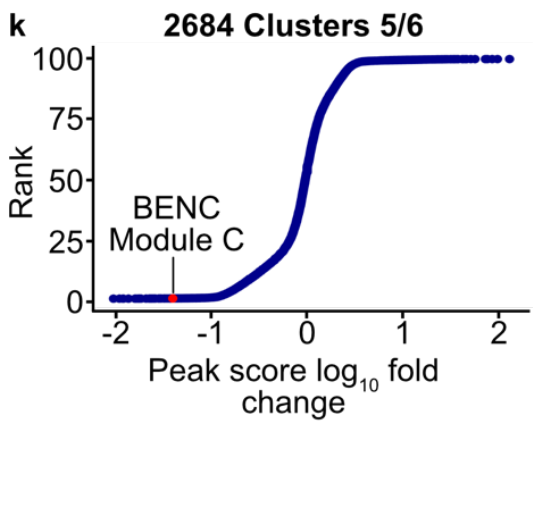

Figure 7. Dual FLT3 and LSD1 inhibition Results in a Shift from a MYC Super-Enhancer-High to a MYC Super-Enhancer-Low Cell State in Primary AML Blasts. 
a-c. Single cell ATAC-seq was performed on three AML patient samples with DMSO or quizartinib (500 nM) and GSK2879552 (500 nM) for 24 hours. UMAP of DMSO and drugtreated cells colored by cluster. d-f. Proportions of DMSO-treated and drug-treated cells assigned to each cluster. Dynamic clusters were identified as the populations that shift between DMSO and drug-treated conditions and with greater accessibility at CD34. Dynamic clusters are highlighted with gray shading between bars. g-i. AUC of accessibility at each BENC module is displayed. j. Treatment group pseudo-bulked accessibility at the MYC BENC modules separated by treatment and patient sample. k. Ranking by $\log _{10}$ (peak score fold change) between DMSO-treated and the combination-treated cells within dynamic clusters. 

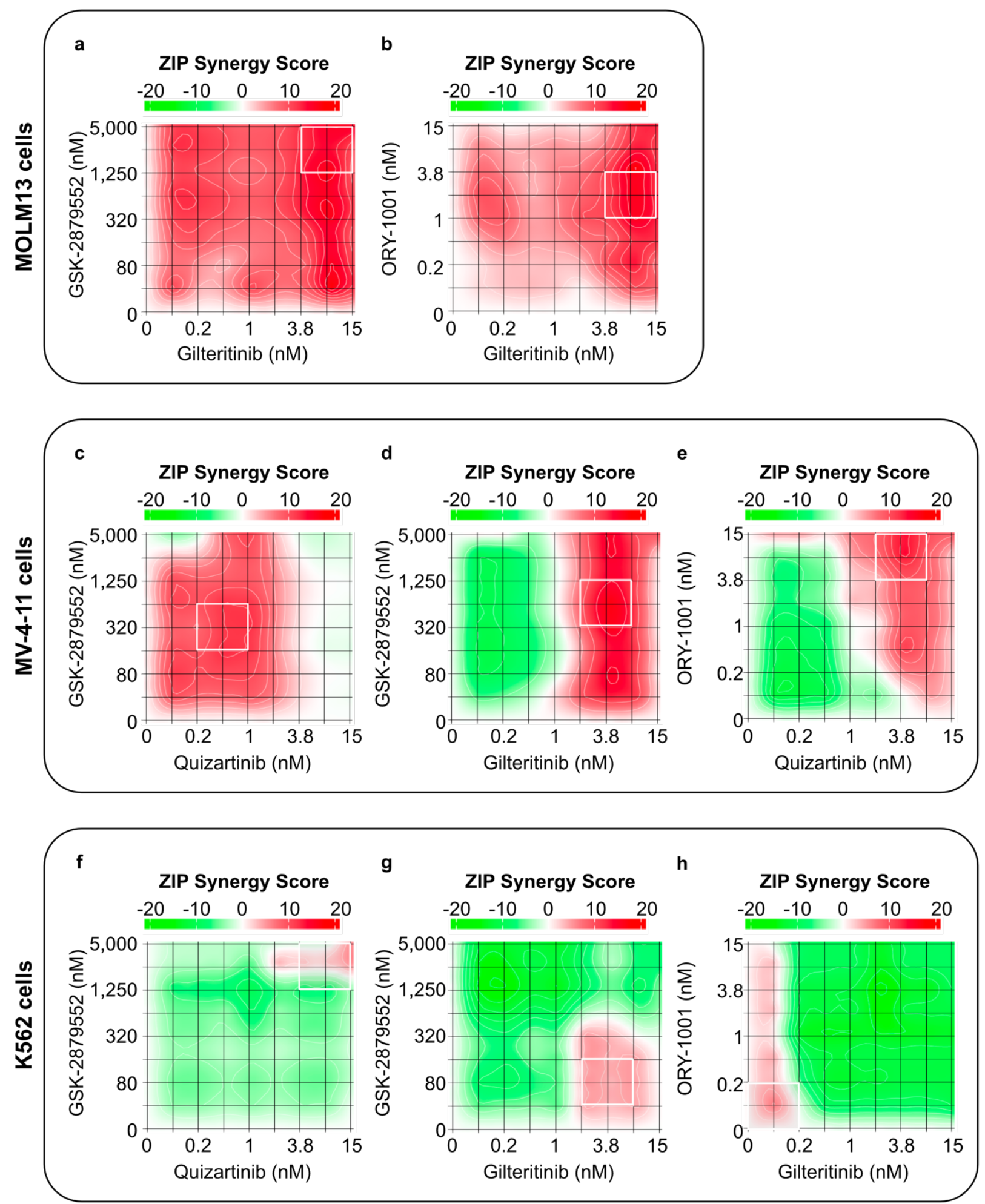

Supplementary Figure 1. Drug Synergy Between FLT3 and LSD1 inhibitors in FLT3mutant and wild type cell lines. a. MOLM13 (FLT3-ITD-positive) cells were treated with an 8X8 matrix of gilteritinib and GSK2879552 or b. gilteritinib and ORY-1001 in triplicate. c. MV4:11 (FLT3-ITD-positive) cells were treated with an 8X8 matrix of quizartinib and 
GSK2879552, d. gilteritinib and GSK2879552, or e. quizartinib and ORY-1001 in triplicate. f. K562 (FLT3-wild-type) cells were treated with an 8X8 matrix of quizartinib and GSK2879552, g. gilteritinib and GSK2879552, or h. gilteritinib and ORY-1001 in triplicate. Cell viability was assessed after 3 days of culture using CellTiter Aqueous colorimetric assay. Synergy was assessed using the ZIP method. 
a Early Apoptosis

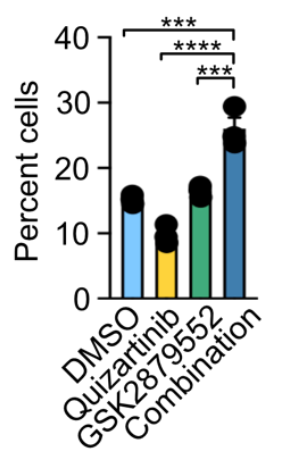

d Gene Expression

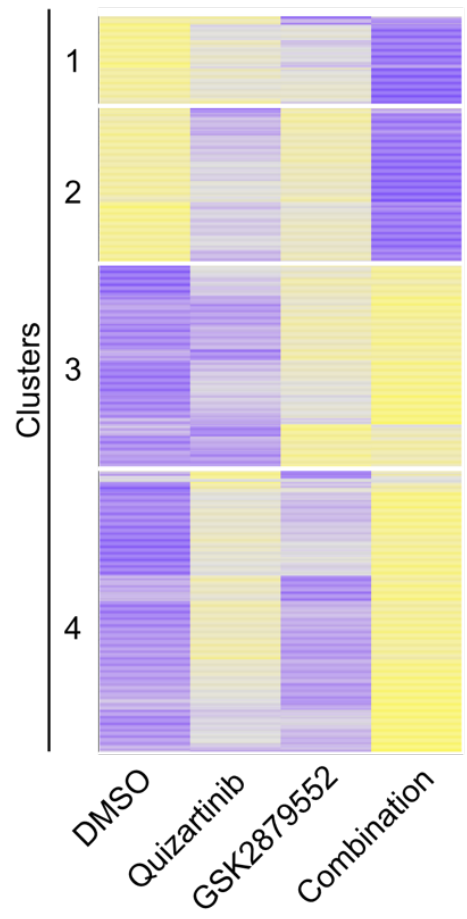

b Late Apoptosis

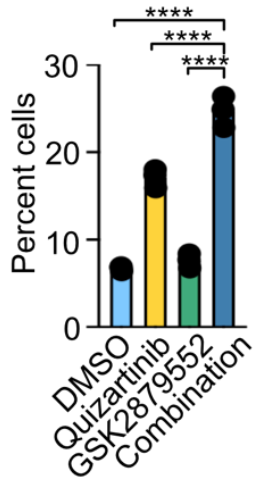

Scaled expression

$\begin{array}{llll}2 & 0 & -2\end{array}$

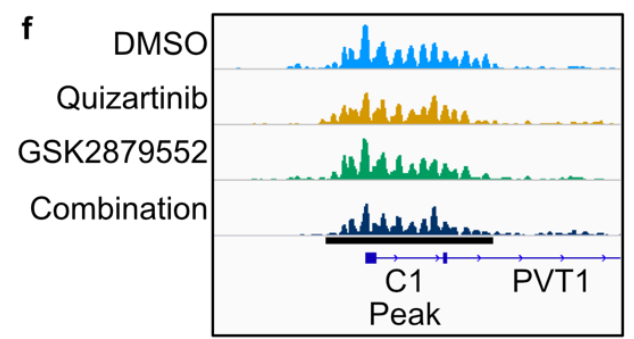

g

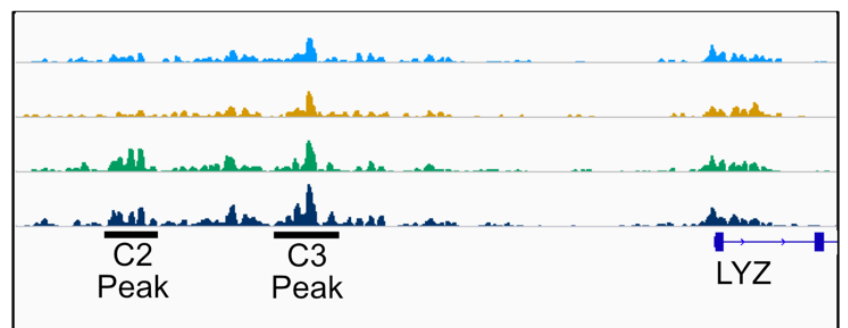

Supplementary Figure 2. Efficacy of Dual FLT3 and LSD1 Inhibition in MOLM13 Cells. a, b. MOLM 13 cells were treated quizartinib (1 nM), GSK2879552 (100 nM), the combination, or an equal volume of DMSO vehicle for 48 hours prior to assessment of apoptosis by flow cytometry. Early apoptosis is Annexin V positive Propidium lodide (PI) 
negative. Late apoptosis is Annexin $V$ positive propidium iodide positive. Statistical significance was determined by two-way ANOVA with a Holm-Šidák post-test correction. c. CD34+ cells from healthy donor marrow were plated in complete human methocult media along with quizartinib (1 nM), GSK2879552 (100 nM) the combination, or an equal volume of DMSO. Colony number was assessed after 14 days of growth. Statistical significance was determined by two-way ANOVA with a Holm-Šidák post-test correction. ns $=$ not significant, ${ }^{*}=p<0.05,{ }^{* *}=p<0.01,{ }^{* * *}=p<0.001,{ }^{* * *}=p<0.0001$. d. Unsupervised hierarchical clustering of differentially expressed genes and e. complete transcription factor target enrichment from RNA-seq on MOLM13 cells performed in Figure 1b. f, g. Example tracks from H3K27Ac CUT\&Tag described in Figure 1d. Examples of different clusters of differential peaks are shown. 

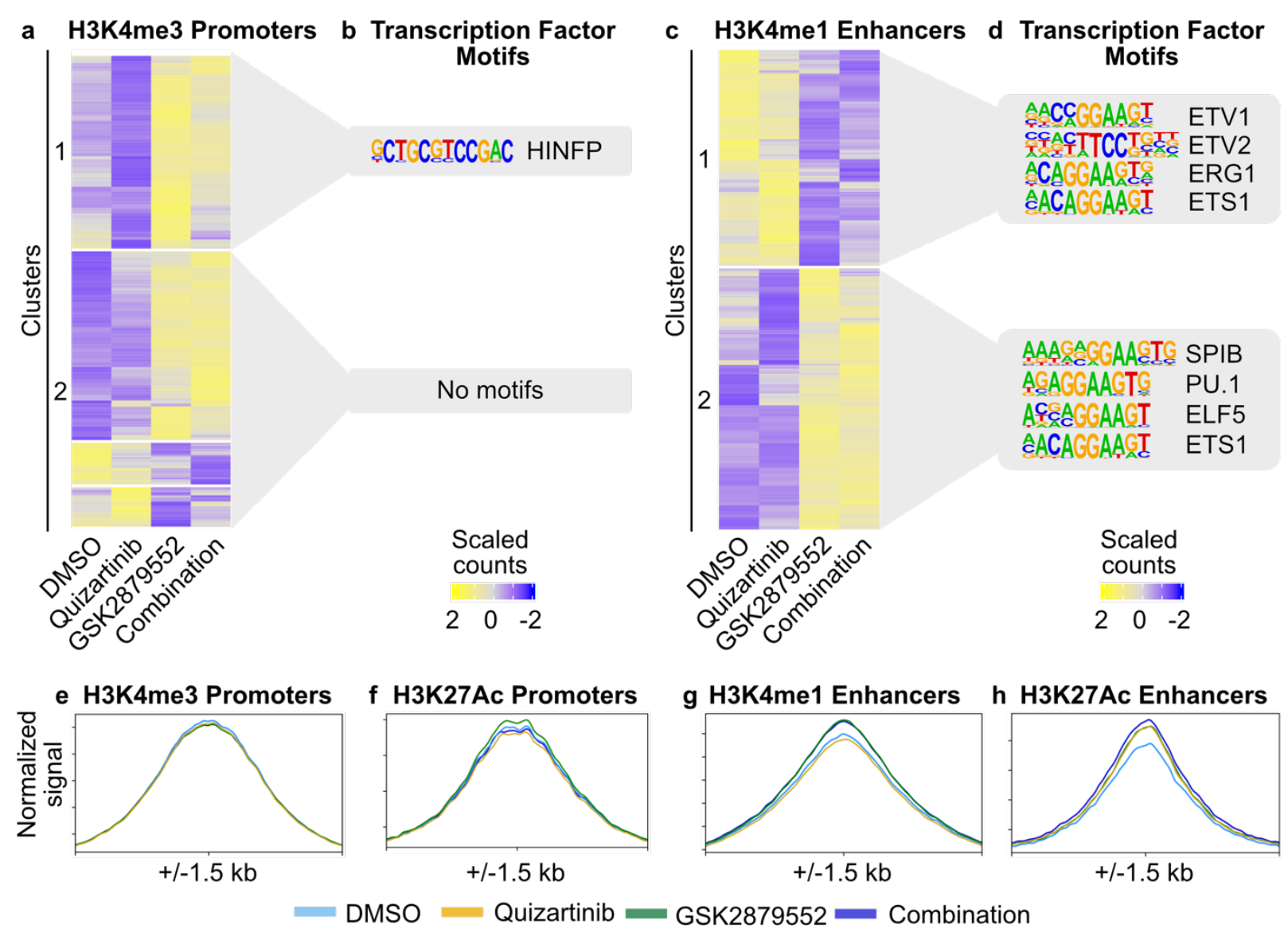

i H3K27Ac Promoter GO Biological Process

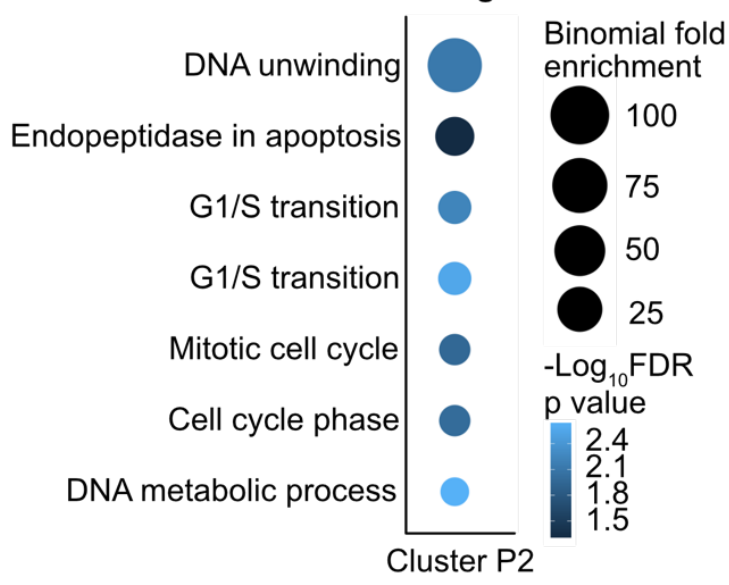

j H3K27Ac Enhancer GO Biological Process

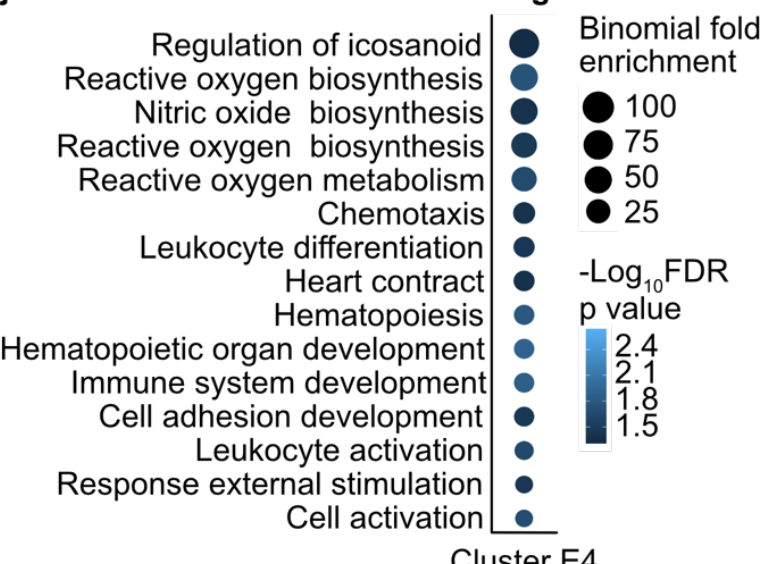

Cluster E4

Supplementary Figure 3. Epigenetic Impact of Dual FLT3 and LSD1 Inhibition. a. Unsupervised hierarchical clustering of regions of differential H3K4me3 at promoters assessed by CUT\&Tag from MOLM13 cells treated with quizartinib (1 nM), GSK2879552 $(100 \mathrm{nM})$, the combination, or an equal volume of DMSO for 6 hours. b. Motif enrichment for regions of differential $\mathrm{H} 3 \mathrm{~K} 4 \mathrm{me} 3$ read density at promoters. Top 4 enriched transcription factor motifs are shown. c. Unsupervised hierarchical clustering of regions of differential H3K4me1 at enhancers assessed by CUT\&Tag from MOLM13 cells treated with quizartinib (1 nM), GSK2879552 (100 nM), the combination, or an equal volume of DMSO 
for 6 hours. d. Motif enrichment for regions of differential H3K4me1 read density at enhancers. Top 4 enriched transcription factor motifs are shown. e. H3K4me3 signal at all promoters assessed by CUT\&Tag in MOLM13 cells treated as above. f. H3K27Ac signal at all promoters assessed by CUT\&Tag in MOLM13 cells treated as above. g. H3K4me1 signal at all enhancers assessed by CUT\&Tag in MOLM13 cells treated as above. $\mathbf{h}$. H3K27Ac signal at all enhancers assessed by CUT\&Tag in MOLM13 cells treated as above. i, j. GO analysis of differential H3K27Ac read pileup at i. promoters and j. enhancers from analysis in Figure 2. Dot size represents the binomial fold enrichment and color represents the $-\log _{10}$ (FDR p-value) of the GO Biological Process term. 

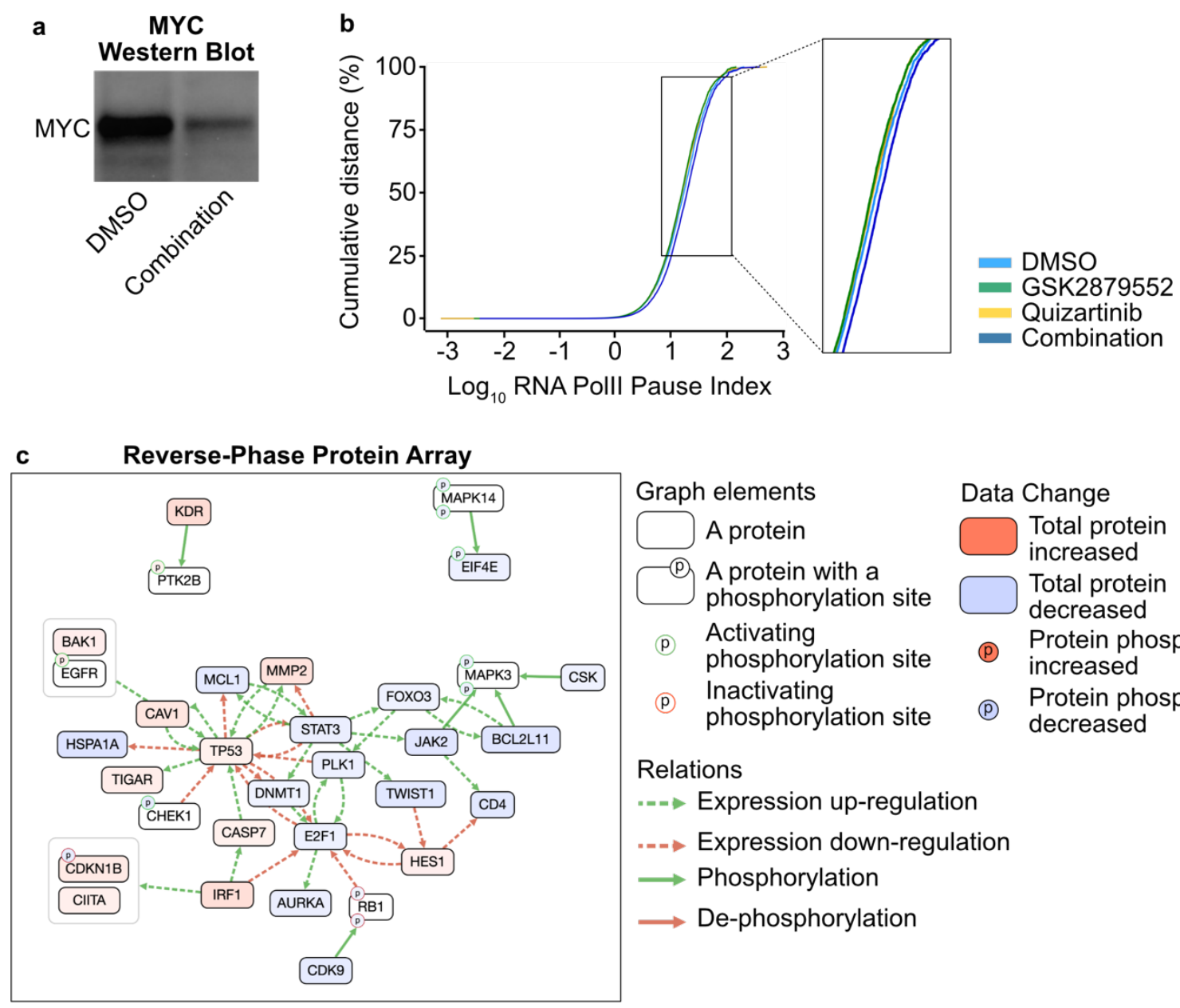

Graph elements
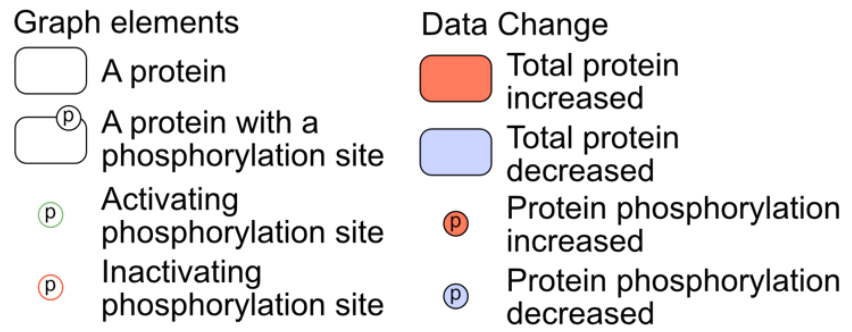

Relations

$\ldots$ Expression up-regulation

$\ldots$ Expression down-regulation

$\longrightarrow$ Phosphorylation

$\longrightarrow$ De-phosphorylation

d MYC Over-Expression

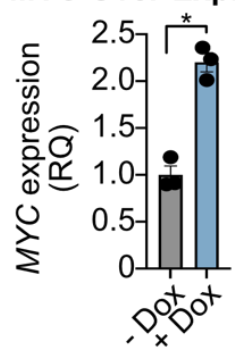

e
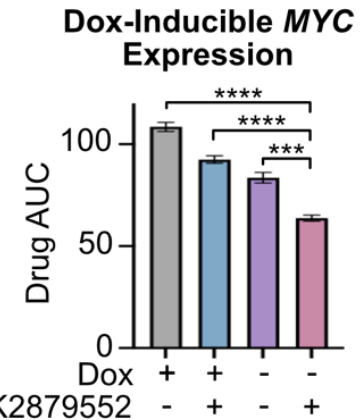

f Dox-inducible MYC Over-Expression Apoptosis

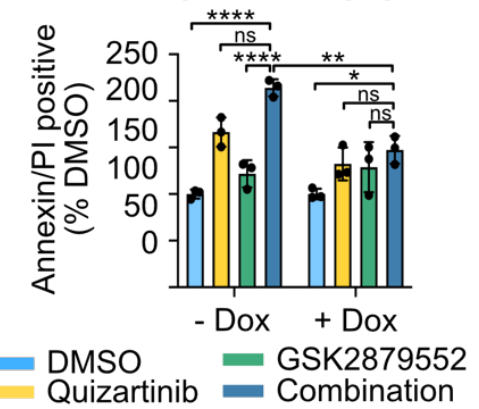

Supplementary Figure 4. Dual FLT3 and LSD1 Inhibition Suppresses MYC Expression and activity. a. Western blot for MYC in MOLM13 cells treated with dual quizartinib $(1 \mathrm{nM})$ and GSK2879552 (100 nM) or an equivalent volume of DMSO for 24 hours. b. MOLM13 cells were treated with quizartinib (1 nM), GSK2879552 (100 nM), the combination, or an equal volume of DMSO vehicle for 6 hours prior to CUT\&Tag for RBP1. Cumulative distribution of RNA Polll pause-indices across all RBP1 and MYC co-bound genes. RNA Polll pause indices within the $25-95 \%$ cumulative distribution, which are depicted in Figure 3c, are highlighted. c. Differential phosphoprotein network enrichment in MOLM13 cells treated for 24 hours with $1 \mathrm{nM}$ quizartinib and $100 \mathrm{nM}$ GSK2979552 or an 
equivalent volume of DMSO. d. MOLM13 cells were transduced with lentiviral particles harboring a doxycycline-inducible MYC expression vector. MYC gene expression was assessed 48 hours after the addition of doxycycline $(1 \mu \mathrm{g} / \mathrm{mL})$ and normalized to GAPDH as an endogenous control. Statistical significance was determined by two-way ANOVA with a Holm-Šidák post-test correction. e. MOLM13 cells were transduced with lentiviral particles harboring a doxycycline-inducible $M Y C$ expression vector. Cells were treated with doxycycline $(1 \mu \mathrm{g} / \mathrm{mL})$ or DMSO for 48 hours and then plated in an 8x8 matrix of quizartinib and GSK2879552 for 3 days prior to viability assessment by CellTiter Aqueous colorimetric assay. AUC data from the $75 \mathrm{nM}$ GSK isoline is shown. Dose responses and synergy over the entire drug matrix is shown in Figure 3. f. MOLM13 cells harboring a doxycycline-inducible MYC construct were treated for 72 hours with doxycycline (1 $\mu \mathrm{g} / \mathrm{mL}$ ) then treated with quizartinib (1 nM), GSK2879552 (100 nM), the combination, or an equal volume of DMSO for 48 hours. Apoptosis was assessed using flow cytometry for Annexin V and PI. ns $=$ not significant, ${ }^{*}=p<0.05,{ }^{* *}=p<0.01,{ }^{* * *}=p<0.001,{ }^{* * *}$ $=p<0.0001$. 

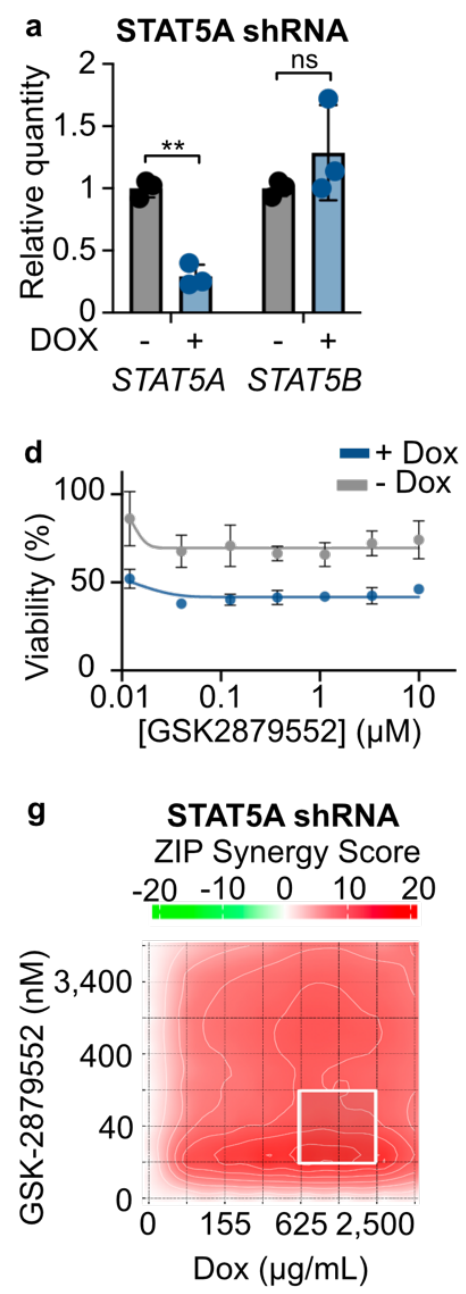

j

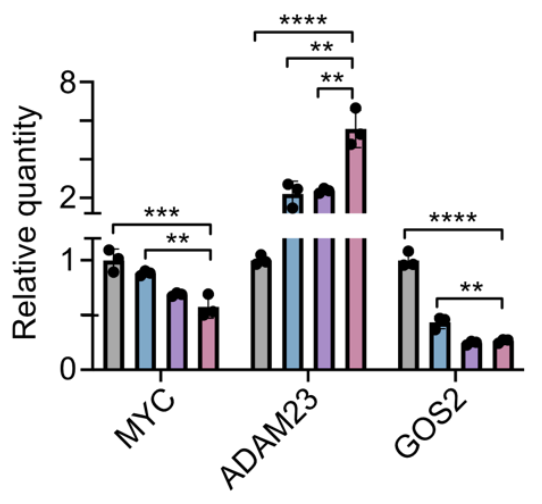

$$
\text { - DMSO Dox - GSK2879552 - Dox + GSK2879552 }
$$

Supplementary Figure 5. STAT5 and MYC Play a Key Role in the Response to FLT3 and LSD1 Inhibition. a-c. MOLM13 cells were transduced with lentiviral particles harboring a doxycycline-inducible STAT5 knockdown. Cells were induced with doxycycline $(1 \mu \mathrm{g} / \mathrm{mL})$ for 48 hours. Validation of knockdown of STAT5A and STA5B by qPCR relative to GUSB endogenous control statistics were performed on the normally distributed dCT

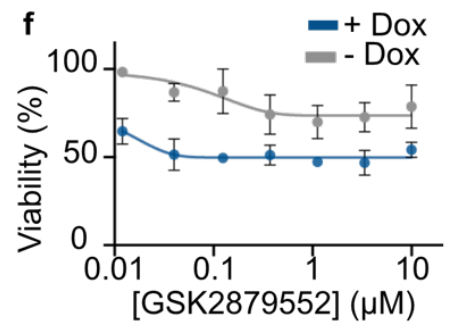

i Dox-Inducible NTC

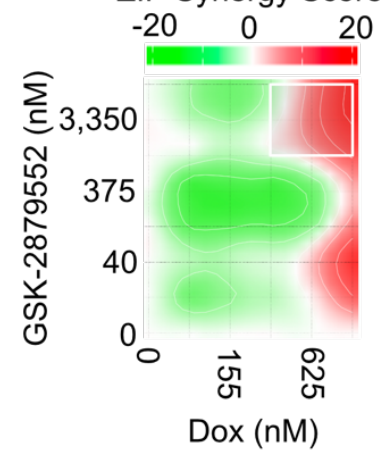

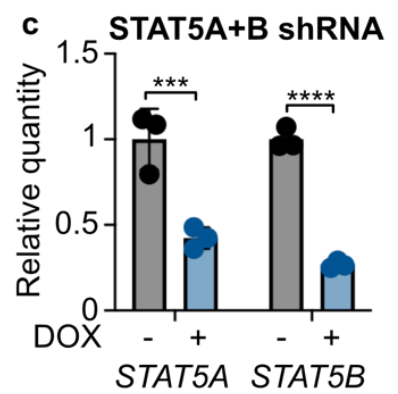

Dox (nM)

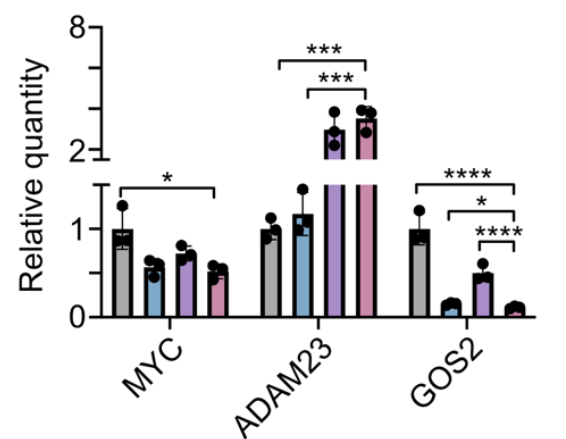

STAT5B shRNA
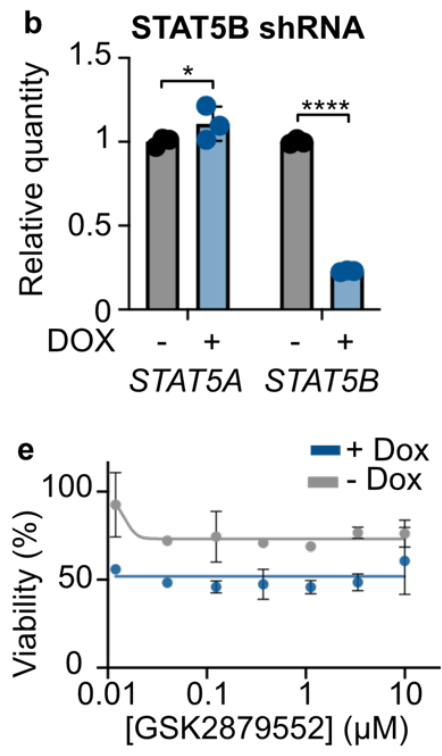

h

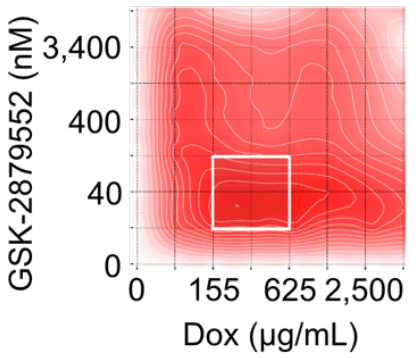

k 
values. Significance was determined by two-way ANOVA with a Holm-Šidák post-test correction. d-f. Dose response curves for GSK2879552 with and without doxycycline (1 $\mu \mathrm{g} / \mathrm{mL})$. In all cases values are represented as the mean +/- SEM. $\mathbf{g}, \mathbf{h}$. 8x8 drug synergy matrices between GSK2879552 and g. STAT5A or h. STAT5B knockdown (induced by doxycycline) were performed in triplicate in MOLM13 cells with viability assessed by CellTiter Aqueous colorimetric assay after 3 days of drug exposure. Zero interaction potency (ZIP) synergy scores were calculated on the average values for each drug dose. $\mathbf{i}$. MOLM13 cells harboring a doxycycline-inducible non-targeting control (NTC) construct were treated doxycycline $(1 \mu \mathrm{g} / \mathrm{mL})$ or an equivalent volume of DMSO for 72 hours then plated in triplicate in an 8X8 matrix of concentrations of GSK2879552 and doxycycline for 3 days. Cell viability was measured using CellTiter Aqueous colorimetric assay. Synergy was assessed using the ZIP method. j, k. qPCR assessment of gene expression in MOLM13 cells expressing a doxycycline-inducible j. STAT5A or k. STAT5B shRNA. Cells were treated with doxycycline $(1 \mu \mathrm{g} / \mathrm{mL})$ or an equivalent volume of DMSO for 72 hours prior to the addition of GSK2879552 (100 nM) for 24 hours. Expression was normalized to GusB as an endogenous control. Statistical significance was determined by two-way ANOVA with a Holm-Šidák post-test correction. ${ }^{*}=p<0.05,{ }^{* *}=p<0.01,{ }^{* * *}=p<$ $0.001,{ }^{* * *}=p<0.0001$. 


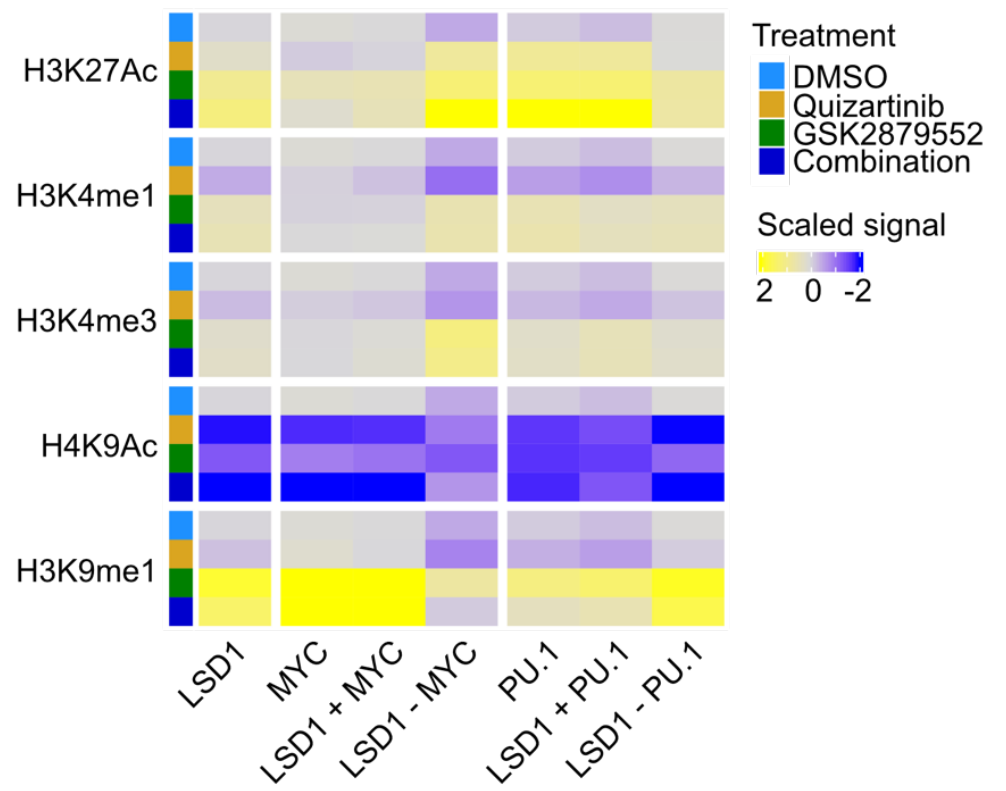

Supplementary Figure 6. PU.1 and MYC Delineate Distinct Sites of LSD1-Dependent Gene Regulation. MOLM13 cells were treated with quizartinib (1 nM), GSK2879552 (100 $\mathrm{nM}$ ), the combination, or an equal volume of DMSO for 6 hours before histone modification assessment. H3K9Ac or H3K9me1 were assessed using CUT\&Tag. Heatmap depiction of normalized read pileup of H3K9Ac and H3K9me1 as well as H3K27Ac, H3K4me1, and H3K4me3 at LSD1 (ChIP-seq), MYC (ChIP-seq) and PU.1 (CUT\&RUN) binding sites (data taken from Figure 2 excluding $\mathrm{H} 3 \mathrm{~K} 9$ marks). 


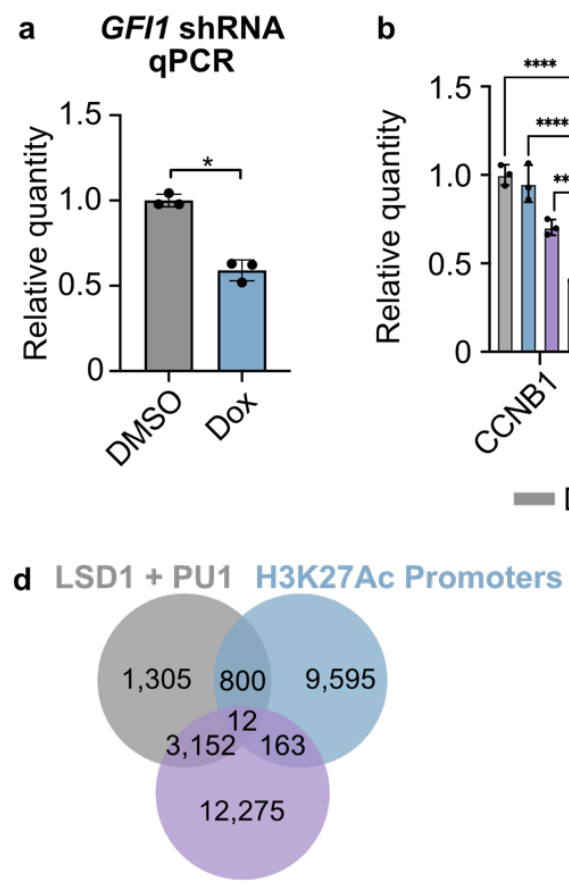

H3K27Ac Enhancers

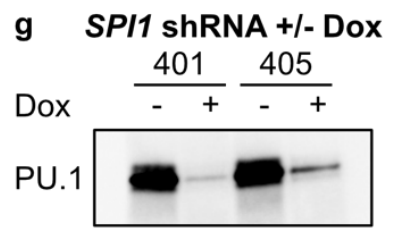

GFI1 ShRNA

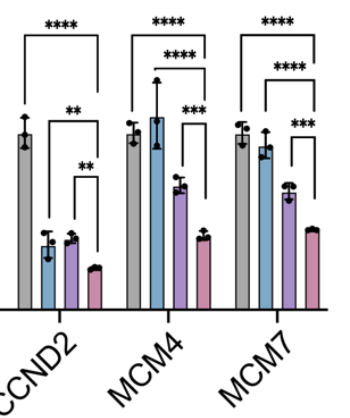

DMSO Dox - GSK2879552

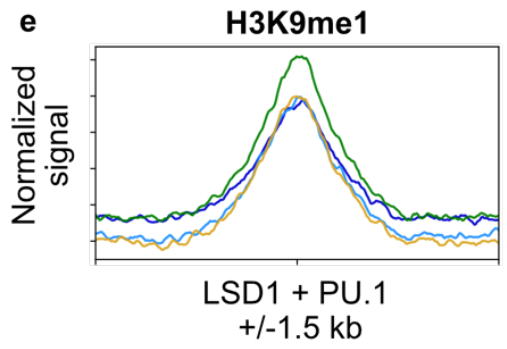

h

SPI1 shRNA 405 -Dox ZIP Synergy Score $\begin{array}{lllll}-40 & -10 & 0 & 10 & 40\end{array}$

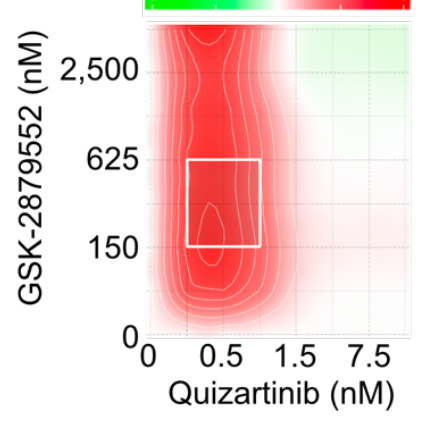

C GFI1 shRNA Inducible NTC

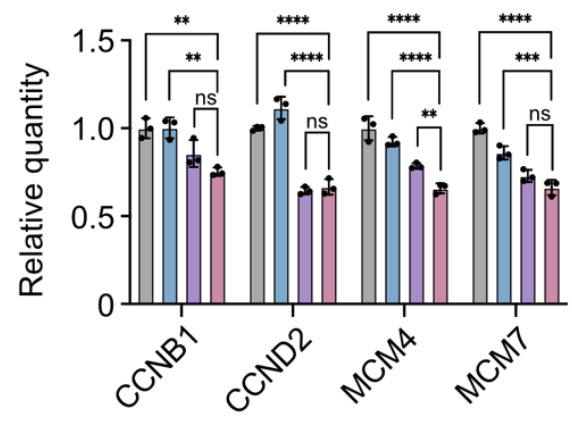

Dox + GSK2879552

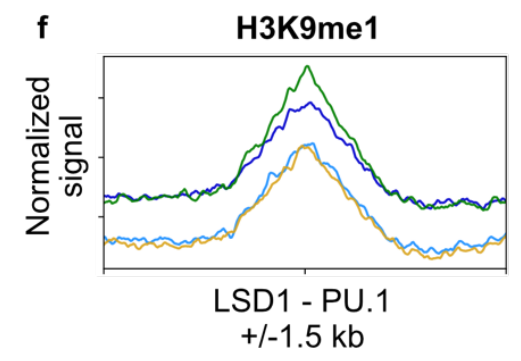

i $\quad$ SPI1 shRNA $405+$ Dox ZIP Synergy Score $\begin{array}{lllll}-30 & -10 & 0 & 10 & 30\end{array}$

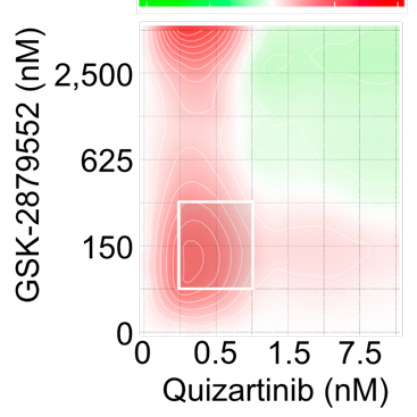

Supplementary Figure 7. Knockdown of SPI1 or GFI1 Weakens the Effects of Dual FLT3 and LSD1 Inhibition. a. Validation of GFI1 knockdown by qPCR relative to GUSB endogenous control. b, c. qPCR assessment of gene expression in MOLM13 cells expressing a doxycycline-inducible b. GFI1 shRNA or c. NTC. Cells were treated with doxycycline $(1 \mu \mathrm{m} / \mathrm{mL})$ for 72 hours prior to the addition of GSK2879552 $(100 \mathrm{nM})$ for 24 hours. Expression was normalized to GusB as an endogenous control. Statistical significance was determined by two-way ANOVA with a Holm-Šidák post-test correction. ns = not significant, ${ }^{*}=p<0.05,{ }^{* *}=p<0.01,{ }^{* * *}=p<0.001,{ }^{* * * *}=p<0.0001$. d. Overlap of LSD1 ChIP-seq and PU.1 CUT\&RUN co-bound peaks with H3K27Ac promoter and enhancer peaks from Figure 2. e, f. Normalized read pileup is shown at LSD1 ChIP-seq peaks that e. do and f. do not overlap with PU.1 CUT\&RUN peaks. g. Western blot for PU.1 in MOLM13 cells stably expressing a doxycycline-inducible SPI1 shRNA treated with doxycycline $(1 \mu \mathrm{g} / \mathrm{ml})$ or an equivalent volume of DMSO for 72 hours. h, i. $8 \times 8$ drug synergy matrices between GSK2879552 and quizartinib with and without PU.1 knockdown (induced by doxycycline) were performed in triplicate in MOLM13 cells with viability 
bioRxiv preprint doi: https://doi.org/10.1101/2022.01.17.476469; this version posted January 18,2022 . The copyright holder for this preprint (which was not certified by peer review) is the author/funder, who has granted bioRxiv a license to display the preprint in perpetuity. It is made available under aCC-BY-NC 4.0 International license.

assessed by CellTiter Aqueous colorimetric assay after 3 days of drug exposure. Zero interaction potency (ZIP) synergy scores were calculated on the average values for each drug dose. 

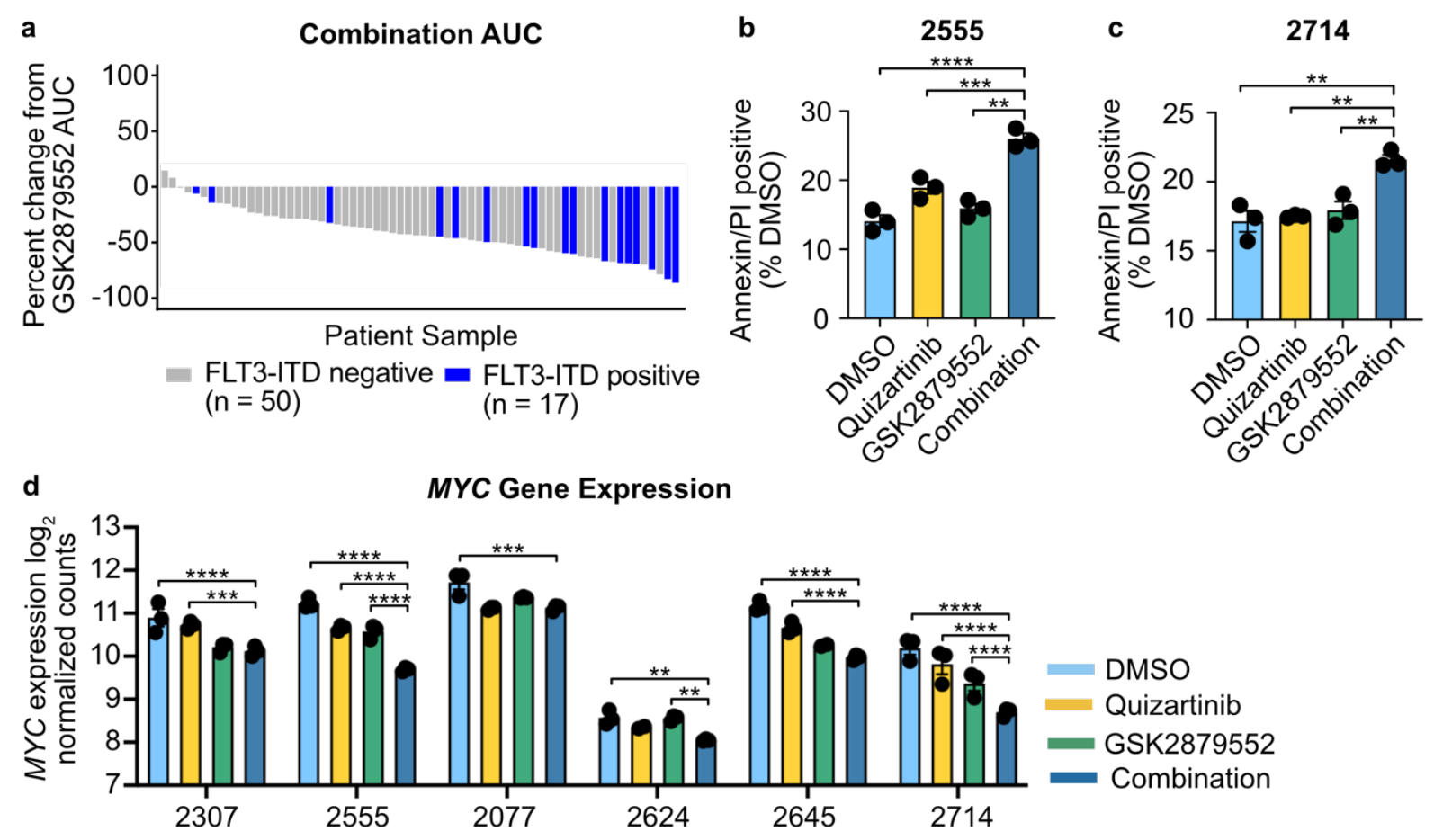

e

MYC Regulon Scores

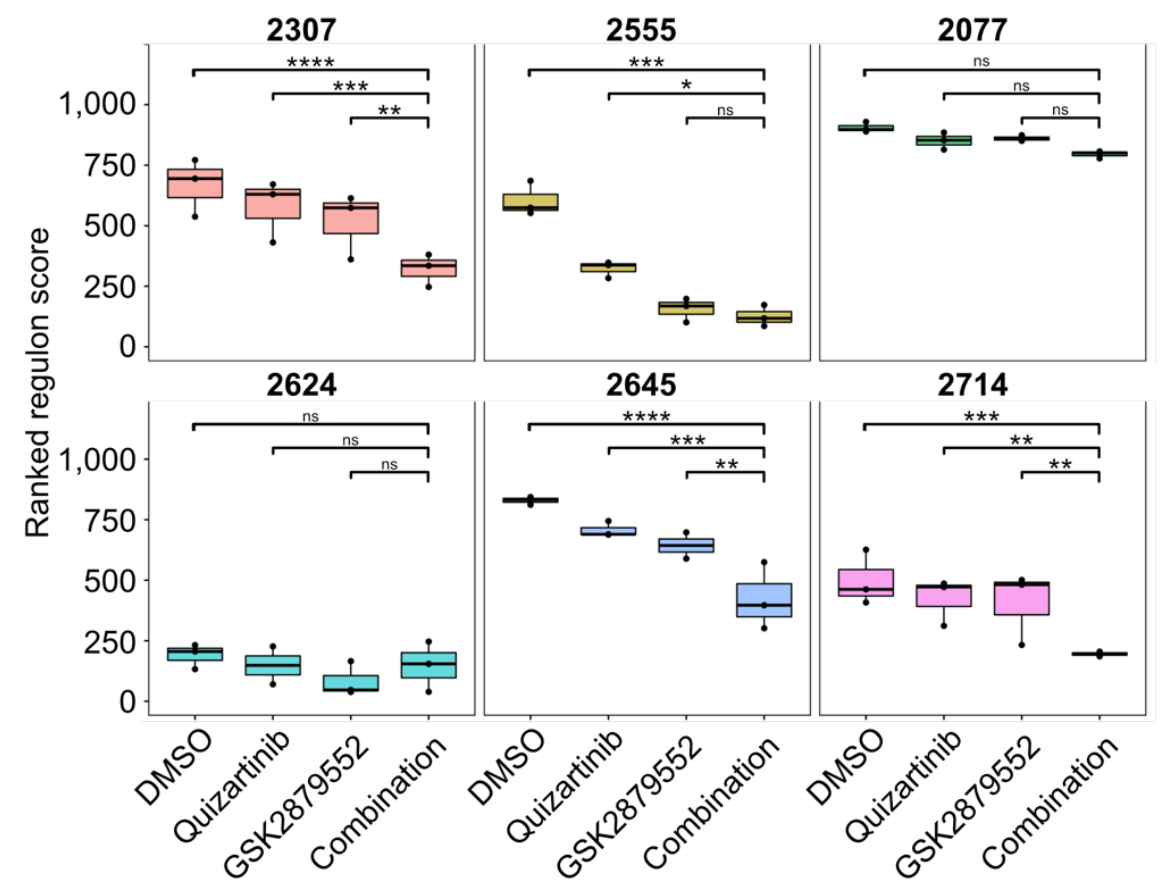

Supplementary Figure 8. Efficacy of Dual FLT3 and LSD1 Inhibition in Primary AML Samples. a. Primary AML blasts from 66 total samples (17 FLT3-ITD-positive) were cultured for 3 days along a 7-point curve with either quizartinib, GSK2879552, or equimolar amounts of the drug combination. Cell viability was assessed by CellTiter Aqueous colorimetric assay. Percent change combination AUC with respect to GSK2879552 for each sample. Bar color indicates FLT3-ITD mutation status. b, c. Primary AML blasts were 
treated with quizartinib (500 nM), GSK2879552 (500 nM), the combination, or an equivalent volume of DMSO for 24 hours. Apoptosis was assessed by flow cytometry for Annexin $\mathrm{V}$ and $\mathrm{PI}$ after 48 hours of drug exposure. d. RNA-seq was performed on six independent, FLT3-ITD-positive patient samples treated in triplicate with $500 \mathrm{nM}$ quizartinib, 500 nM GSK2879552, both drugs in combination, or an equivalent volume of DMSO for 24 hours. MYC gene expression in each individual sample is shown. e. Ranking of normalized regulon enrichment scores on RNA-seq in d. Statistical significance was determined by two-way ANOVA with a Holm-Šidák post-test correction. ns = not significant, * $=p<0.05,{ }^{* *}=p<0.01,{ }^{* * *}=p<0.001,{ }^{* * *}=p<0.0001$. 

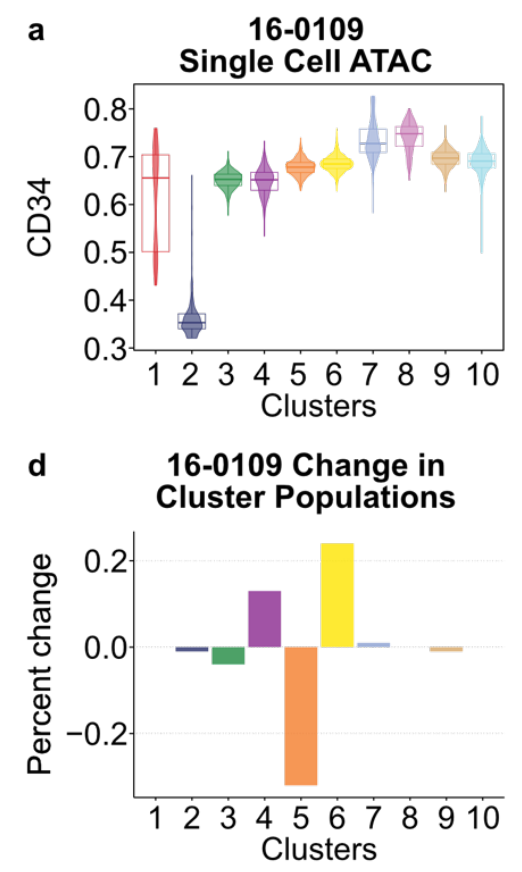

b

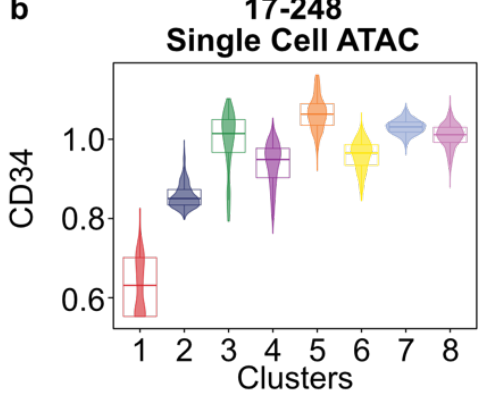

e

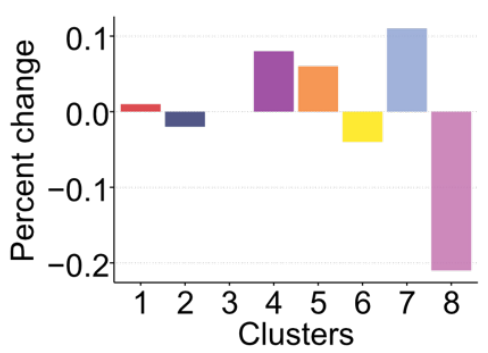

C

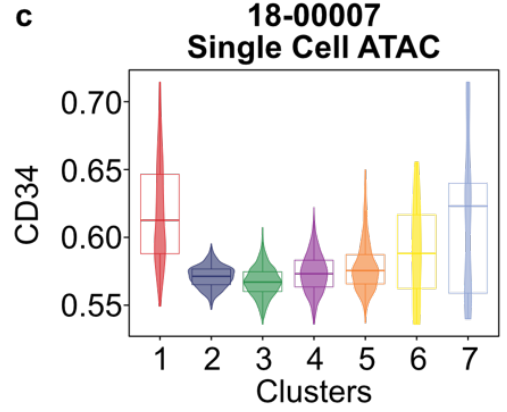

f

18-00007 Change in Cluster Populations

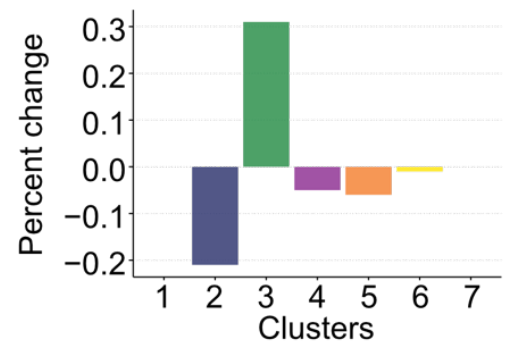

Supplementary Figure 9. Marker for immature populations in primary patient sample single cell ATAC-seq. a-c. Primary AML blasts from three FLT3-ITD-positive samples MOLM13 cells were treated with quizartinib (500 nM) and GSK2879552 (500 nM) or an equal volume of DMSO vehicle for 24 hours prior to single cell ATAC sequencing. Clusters contain cell from both conditions. The CD34 gene scores were calculated from chromatin accessibility and are an estimate of gene transcription. d-f. Percent change in cluster populations between DMSO and combination treatments. 